\title{
CONVEX SUBCONES OF THE CONTINGENT CONE IN NONSMOOTH CALCULUS AND OPTIMIZATION
}

\author{
DOUG WARD
}

\begin{abstract}
The tangential approximants most useful in nonsmooth analysis and optimization are those which lie "between" the Clarke tangent cone and the Bouligand contigent cone. A study of this class of tangent cones is undertaken here. It is shown that although no convex subcone of the contingent cone has the isotonicity property of the contingent cone, there are such convex subcones which are more "accurate" approximants than the Clarke tangent cone and possess an associated subdifferential calculus that is equally strong. In addition, a large class of convex subcones of the contingent cone can replace the Clarke tangent cone in necessary optimality conditions for a nonsmooth mathematical program. However, the Clarke tangent cone plays an essential role in the hypotheses under which these calculus rules and optimality conditions are proven. Overall, the results obtained here suggest that the most complete theory of nonsmooth analysis combines a number of different tangent cones.
\end{abstract}

1. Introduction. Research in convex and nonsmooth analysis has, over the past quarter century, considerably broadened the scope of optimization theory. Indeed, optimization theory has grown during this period to encompass, successively, problems involving

(i) convex functions [24].

(ii) locally Lipschitzian functions $[\mathbf{5}, \mathbf{1 1}]$.

(iii) certain classes of locally lower semicontinuous functions $[\mathbf{2 5}, \mathbf{5}, \mathbf{1 5}, \mathbf{1}, \mathbf{2 8}$, 33].

The analysis developed for stages (ii) and (iii) centers around local approximations to sets called tangent cones. A plethora of these tangential approximants have been defined (see for instance $[6,18,19,30,22,32]$ ), of which a few have proven to be particularly useful. We review below the definitions of three of them. Here and throughout the paper, $E$ will denote a Banach space.

DEFINITION 1.1. Let $C \subset E$ and $x_{0}$ an element of the closure of $C$ (hereafter denoted $\operatorname{cl} C$ ).

(a) The contingent cone to $C$ at $x_{0}$ is the set

$$
K_{C}\left(x_{0}\right):=\left\{y \in E \mid \exists t_{k} \downarrow 0, \exists y_{k} \rightarrow y, x_{0}+t_{k} y_{k} \in C\right\} .
$$

(b) The Ursescu tangent cone to $C$ at $x_{0}$ is the set

$$
k_{C}\left(x_{0}\right):=\left\{y \in E \mid \forall t_{k} \downarrow 0, \exists y_{k} \rightarrow y, x_{0}+t_{k} y_{k} \in C\right\} .
$$

Received by the editors June 20, 1986.

1980 Mathematics Subject Classification (1985 Revision). Primary 58C20; Secondary 90C30, $46 \mathrm{G} 05$.

Key words and phrases. Clarke tangent cone, contingent cone, subgradient, Liusternik theorem, strong general position. 
(c) The Clarke tangent cone to $C$ at $x_{0}$ is the set

$$
T_{C}\left(x_{0}\right):=\left\{y \in E \mid \forall x_{k} \rightarrow x_{0} \text { with } x_{k} \in C, \forall t_{k} \downarrow 0, \exists y_{k} \rightarrow y, x_{k}+t_{k} y_{k} \in C\right\} .
$$

It follows from Definition 1.1 that each of these cones is always a closed set, and that the inclusions

$$
T_{C}\left(x_{0}\right) \subset k_{C}\left(x_{0}\right) \subset K_{C}\left(x_{0}\right)
$$

are true in general. The Ursescu tangent cone, perhaps the least well known of the three, has received increasing attention in recent years $[30,6,19,7,8,33$, 22]. Each of these cones has an alternate definition valid in any locally convex topological vector space $[\mathbf{2 5}, \mathbf{3 0}]$, but since the main results to be presented here are Banach space results, we will conduct our entire discussion in a Banach space setting.

Each of these tangent cones has strengths and weaknesses. For example, the contingent cone is isotone with respect to inclusion; i.e.,

$$
K_{C}\left(x_{0}\right) \subset K_{D}\left(x_{0}\right) \quad \text { whenever } C \subset D .
$$

A rudimentary theory of necessary optimality conditions can be built upon this property $[\mathbf{3 1}, \mathbf{3 4}]$. The Ursescu tangent cone is also isotone, but the Clarke tangent cone is not ([31]; see also Theorem 1.2 below).

On the other hand, the Clarke tangent cone is always a convex cone $[\mathbf{1 9}, \mathbf{2 6}, \mathbf{5}$, 6] and is thus a powerful analytical tool. The contingent and Ursescu tangent cones are not always convex, however, a fact that somewhat restricts their usefulness.

One can construct a closed, convex, isotone tangent cone by taking the closed convex hull of the contingent cone. The resulting object, called the pseudotangent cone, is useful in differentiable programming [10]; however, it is too "large" to play a corresponding role in nonsmooth optimization where convex subcones of the contingent cone become important.

In this paper, we investigate the convex cones $A$ which satisfy the inclusions

$$
T_{C}\left(x_{0}\right) \subset A_{C}\left(x_{0}\right) \subset K_{C}\left(x_{0}\right) .
$$

The preceding paragraphs suggest that we begin with the following question:

(Q1) Is there some "sensible" tangent cone satisfying (1.2) which is both convex and isotone?

This question has a definite negative answer, as we now demonstrate. In the statement of this theorem, we denote by $P\left(1 \mathbf{R}^{n}\right)$ the power set of $1 \mathbf{R}^{\eta}$.

THEOREM 1.2. There is no mapping $A: \mathcal{P}\left(1 \mathbf{R}^{n}\right) \times 1 \mathbf{R}^{n} \rightarrow \mathcal{P}\left(1 \mathbf{R}^{n}\right)$ which has all of the following properties:

(a) $A$ is isotone.

(b) $A$ is convex.

(c) $A\left(C, x_{0}\right) \subset K_{C}\left(x_{0}\right)$ for all $C \subset 1 \mathbf{R}^{n}$ and $x_{0} \in \mathrm{cl} C$.

(d) $A\left(C, x_{0}\right) \supset C$ whenever $C$ is a one-dimensional subspace of $1 \mathbf{R}^{n}$ and $x_{0} \in C$.

Proof. Consider the subsets of $1 \mathbf{R}^{n}$ defined by $C_{1}: 1 \mathbf{R} \times\{0\}$ and $C_{2}:=$ $\{0\} \times 1 \mathrm{R}$. If $A$ has property (c), $A\left(C_{1} \cup C_{2},(0,0)\right) \subset C_{1} \cup C_{2}$. On the other hand, if $A$ has properties (d) and (a), $C_{1} \cup C_{2} \subset A\left(C_{1}, 0\right) \cup A\left(C_{2}, 0\right)=A\left(C_{1} \cup C_{2},(0,0)\right)$. Thus $A\left(C_{1} \cup C_{2},(0,0)\right)=C_{1} \cup C_{2}$ if $A$ has properties (a), (c) and (d). Such an $A$ cannot have property (b). 
REMARK 1.3. (a) One can easily find mappings $A$ which possess three of the properties listed in Theorem 1.2. All tangent cones satisfying the first inclusion of (1.2) also satisfy (d) of Theorem 1.2.

(b) A number of other "tangent cone impossibility theorems" are collected in [32].

Given a negative answer to (Q1), we shift our attention to a broader question:

(Q2) Are there convex cones satisfying (1.2) which are more accurate approximants than $T$ and possess the analytical strengths of $T$ ?

We will give a qualified affirmative answer to (Q2). Specifically, we show that two particular convex tangent cones satisfying (1.2) have an associated subdifferential calculus as extensive as that for the Clarke tangent cone. In addition, we demonstrate that large classes of convex subcones of $K$ and $k$ can replace $T$ in necessary conditions for optimality in nonsmooth mathematical programming. We hasten to add, however, that these results seem to require assumptions involving the Clarke tangent cone. Our theorems and examples indicate that the Clarke tangent cone plays a special role in nonsmooth analysis.

Here is an outline of the remainder of the paper: In $\S 2$, we define and examine some basic properties of three convex tangent cones satisfying (1.2). In $\S 3$, we review a Liusternik-type theorem which enables us to prove key tangent cone inclusions. We present in $\S 4$ a sort of "algorithm" for generating subdifferential calculus formulae. This procedure, which reduces the proofs of calculus rules to the verification that a tangent cone has three specific properties, was used quite successfully in [33]. We apply this algorithm in $\S 5$ to establish a calculus for the directional derivatives and subgradients associated with the tangent cones discussed in $\S 2$. In $\S 6$ we apply our directional derivative calculus formulae to derive necesary optimality conditions for a nonsmooth mathematical program. These conditons sharpen, in a Banach space setting, optimality conditions given in [25, 33, and 21].

At this juncture we compile a list of notations used in this paper. For an extended-real-valued function $f: E \rightarrow \overline{1 \mathbf{R}}$, we denote by epi $f$ the epigraph of $f$. By the domain of $f$, we mean the set

$$
\operatorname{dom} f:=\{x \in E \mid f(x)<+\infty\} .
$$

We say that $f$ is proper if $\operatorname{dom} f$ is nonempty and $f$ never takes on the value $-\infty$. If $f$ is convex, $\partial f\left(x_{0}\right)$ will denote the subgradient of $f$ at $x_{0}[\mathbf{2 4}]$.

We say that a mapping $A: \mathcal{P}(E) \times E \rightarrow \mathcal{P}(E)$ is a tangent cone if $A\left(C, x_{0}\right)$ (which we will usually write $A_{C}\left(x_{0}\right)$ ) is a (possibly empty) cone for all nonempty $C \subset E$ and $x_{0} \in \operatorname{cl} C$. As in Theorem 1.2 , we will say that $A$ has a certain property if $A_{C}\left(x_{0}\right)$ has that property for all nonempty $C \subset E$ and $x_{0} \in \operatorname{cl} C$. For two tangent cones $A$ and $A^{\prime}$, we say $A^{\prime} \subset A$ if the inclusion $A_{C}^{\prime}\left(x_{0}\right) \subset A_{C}\left(x_{0}\right)$ is true for all nonempty $C \subset E$ and $x_{0} \in \operatorname{cl} C$. We will denote by $A_{f}\left(x_{0}\right)$ the set $A_{\text {epi } f}\left(x_{0}, f\left(x_{0}\right)\right)$.

We denote the dual space of a Banach space $E$ by $E^{\prime}$. For $\delta>0$, we define

$$
N_{\delta}(x):=\{y \in E \mid\|x-y\|<\delta\} .
$$

For a nonempty set $C \subset E$, we denote the interior of $C$ by int $C$. By the recession cone of $C$, we mean the set

$$
0^{+} C:=\{y \in E \mid C+y \subset C\}
$$


by the polar of $C$, the set

$$
C^{0}=\{x \in E \mid\langle x, y\rangle \leq 0 \text { for all } y \in C\}
$$

and by the indicator function of $C$, we mean the function $i_{C}: E \rightarrow \overline{1 \mathbf{R}}$ defined by

$$
i_{C}(x)= \begin{cases}0 & \text { if } x \in C, \\ +\infty & \text { else. }\end{cases}
$$

We denote the nonnegative orthant in $1 \mathbf{R}^{n}$ by $1 \mathbf{R}_{+}^{n}$.

2. Some convex tangent cones. In this section we discuss three convex tangent cones which satisfy (1.2) and have recently been studied by Penot [22], Frankowska $[\mathbf{7}, 8]$, and others $[\mathbf{9}, \mathbf{2 0}, \mathbf{2 9}]$.

To begin, we observe that one way to produce a closed convex cone is to take the recession cone of a closed cone. Let us then define, for $C \subset E$ and $x_{0} \in \operatorname{cl} C$, the closed convex tangent cones

$$
\begin{aligned}
& K_{C}^{\infty}\left(x_{0}\right):=\left\{y \in E \mid K_{C}\left(x_{0}\right)+y \subset K_{C}\left(x_{0}\right)\right\}, \\
& k_{C}^{\infty}\left(x_{0}\right):=\left\{y \in E \mid k_{C}\left(x_{0}\right)+y \subset k_{C}\left(x_{0}\right)\right\} .
\end{aligned}
$$

It follows readily from (2.1) and (2.2) that $T \subset K^{\infty} \subset K$ and $T \subset k^{\infty} \subset k$ [19, Theorems 1, 2]. An example of $C$ and $x_{0}$ for which $T_{C}\left(x_{0}\right), K_{C}^{\infty}\left(x_{0}\right)$, and $k_{C}^{\infty}\left(x_{0}\right)$ are distinct is given in [19]. Interestingly, $k^{\infty}$ is not always contained in $K^{\infty}$, even though $k \subset K$. For example, define $f: 1 \mathbf{R} \rightarrow 1 \mathbf{R}$ by

$$
f(x):= \begin{cases}0 & \text { if } x=0, \\ -2^{-(n+1)} & \text { if } 2^{-(n+1)} \leq|x|<2^{-n}, \quad n=0, \pm 1, \pm 2, \ldots,\end{cases}
$$

and let $C:=\operatorname{epi} f$ and $x_{0}:=(0,0)$. Here

$$
\begin{aligned}
K_{C}\left(x_{0}\right) & =\{(x, y)|y \geq-| x \mid\}, \\
k_{C}\left(x_{0}\right) & =\{(x, y)|y \geq-| x \mid / 2\},
\end{aligned}
$$

so that

$$
\begin{aligned}
K_{C}^{\infty}\left(x_{0}\right) & =\{(x, y)|y \geq| x \mid\}, \\
k_{C}^{\infty}\left(x_{0}\right) & =\{(x, y)|y \geq| x \mid / 2\} .
\end{aligned}
$$

As we will see presently, $k^{\infty}$ is somewhat easier to work with than $K^{\infty}$ is, and it has received more attention in the literature $[\mathbf{7}, \mathbf{8}, \mathbf{2 2}]$. In particular, Frankowska has applied $k^{\infty}$ in the study of a general Bolza problem in the calculus of variations [8].

In [22], Penot gives an interesting alternate definition of $k^{\infty}$ :

$$
\begin{aligned}
k_{C}^{\infty}\left(x_{0}\right)=\left\{y \mid \forall\left(x_{n}, t_{n}\right) \rightarrow\left(x_{0}, 0^{+}\right), \forall z\right. & \in k_{C}\left(x_{0}\right) \text { with } \\
x_{n} \in C, t_{n}^{-1}\left(x_{n}-x_{0}\right) & \left.\rightarrow z, \exists y_{n} \rightarrow y, x_{n}+t_{n} y_{n} \in C\right\} .
\end{aligned}
$$

Equation (2.3) makes clear the fact that $T \subset k^{\infty}$. It also suggests the experiment of replacing " $z \in k_{C}\left(x_{0}\right)$ " in (2.3) with " $z \in S$ " for others sets $S$, and studying the resulting objects. Of course the larger the set $S$, the smaller the object obtained. For example, the choice $S:=T_{C}\left(x_{0}\right)$ gives a cone which always contains $k^{\infty}$. This cone is not necessarily convex, however. On the other hand, the choice $S:=K_{C}\left(x_{0}\right)$ gives a convex tangent cone which is always contained in $k^{\infty}$. When $\left\{x_{n}\right\} \subset$ $C,\left\{t_{n}^{-1}\left(x_{n}-x_{0}\right)\right\}$ converges if and only if it converges to an element of $K_{C}\left(x_{0}\right)$, so 
$S:=E$ gives the same cone as $S:=K_{C}\left(x_{0}\right)$. The resulting tangent cone is Penot's prototangent cone

$$
\begin{array}{r}
P_{C}\left(x_{0}\right):=\left\{y \mid \forall\left(x_{n}, t_{n}\right) \rightarrow\left(x_{0}, 0^{+}\right) \text {with } x_{n} \in C \text { and } t_{n}^{-1}\left(x_{n}-x_{0}\right)\right. \\
\text { convergent, } \left.\exists y_{n} \rightarrow y, x_{n}+t_{n} y_{n} \in C\right\} .
\end{array}
$$

If follows from (2.4) that $T \subset P \subset k^{\infty} \subset k \subset K$, with $P_{C}\left(x_{0}\right)=k_{C}^{\infty}\left(x_{0}\right)=K_{C}^{\infty}\left(x_{0}\right)$ whenever $k_{C}\left(x_{0}\right)=K_{C}\left(x_{0}\right)$.

REMARK 2.1. (a) In a forthcoming paper [29], Treiman defines a tangent cone in Banach space which reduces to $P$ in the finite-dimensional case and whose polar cone, like that of $T$, has a useful characterization in terms of "proximal normals".

(b) In (2.3) and (2.4), one may replace " $x_{n}+t_{n} y_{n} \in C$ " with " $x_{\sigma(n)}+t_{\sigma(n)} y_{\sigma(n)} \in$ $C$ for some subsequence $\left\{x_{\sigma(n)}+t_{\sigma(n)} y_{\sigma(n)}\right\}$ ".

An analogous statement is true for $T[\mathbf{1 2}]$ and $k$.

In $\S \S 4$ and 5 , we will be particularly concerned with two questions for each of the tangent cones $A$ that we have defined:

(a) Does the inclusion

$$
A_{C}\left(x_{0}\right) \times A_{D}\left(y_{0}\right) \subset A_{C \times D}\left(x_{0}, y_{0}\right)
$$

hold in general?

(b) For what linear mappings $M: E \rightarrow E_{1}$ and $\left(C, z_{0}\right) \subset \mathcal{P}(E) \times E$ does the inclusion

$$
M\left(A_{C}\left(z_{0}\right)\right) \subset A_{M(C)}\left(M z_{0}\right)
$$

hold?

We give below the answer to question (a). The proofs, which are completely straightforward, are left to the reader.

Proposition 2.2. Let $C$ and $D$ be nonempty subsets of $E$ and $E_{1}$, respectively, and let $x_{0} \in \operatorname{cl} C$ and $y_{0} \in \operatorname{cl} C$. Then

$$
\begin{aligned}
& k_{C}\left(x_{0}\right) \times k_{D}\left(y_{0}\right)=k_{C \times D}\left(x_{0}, y_{0}\right) . \\
& T_{C}\left(x_{0}\right) \times T_{D}\left(y_{0}\right)=T_{C \times D}\left(x_{0}, y_{0}\right) . \\
& k_{C}^{\infty}\left(x_{0}\right) \times k_{D}^{\infty}\left(y_{0}\right)=k_{C \times D}^{\infty}\left(x_{0}, y_{0}\right) . \\
& P_{C}\left(x_{0}\right) \times P_{D}\left(y_{0}\right) \subset P_{C \times D}\left(x_{0}, y_{0}\right), \\
& K_{C}\left(x_{0}\right) \times k_{D}\left(y_{0}\right) \subset K_{C \times D}\left(x_{0}, y_{0}\right) .
\end{aligned}
$$

It is not possible to combine $K^{\infty}$ with any of the other tangent cones above to produce an analogue of $(2.11)$, a defect of $K^{\infty}$ which will limit its usefulness in the sequel. For example, define

$$
\begin{aligned}
& C:=\left\{x \in 1 \mathbf{R} \mid x=2^{-2 n}, n=1,2,3, \ldots\right\} \cup\{0\}, \\
& D:=\left\{x \in 1 \mathbf{R} \mid x=2^{-2 n+1}, n=1,2,3, \ldots\right\} \cup\{0\},
\end{aligned}
$$

and let $\left(x_{0}, y_{0}\right)=(0,0)$. Then $K_{C}(0)=K_{D}(0)=1 \mathbf{R}_{+}$, while $k_{C}(0)=k_{D}(0)=\{0\}$ and $K_{C \times D}^{\infty}\left(x_{0}, y\right)=\{(0,0)\}$ (see [2]). As a result, the inclusion $K_{C}^{\infty}(0) \times A_{D}(0) \subset$ $K_{C \times D}^{\infty}(0,0)$ is not true for $A=K, K^{\infty}, k, k^{\infty}, P$, or $T$.

The cones $K$ and $k$ satisfy (2.6) for any nonempty $C, x_{0} \in \operatorname{cl} C$, and continuous linear $M$ (see for example [33]). Conditions under which $T$ satisfies (2.6) are given in $\left[2\right.$ and 33]. We now present conditions sufficient to give (2.6) for $k^{\infty}, K^{\infty}$, and $P$. 
LEMMA 2.3. Let $C \subset E$ be nonempty with $z_{0} \in \operatorname{cl} C$, and let $M: E \rightarrow E_{1}$ be linear and continuous. The following implications hold:

(1) If $k_{M(C)}\left(M z_{0}\right) \subset M\left(k_{C}\left(z_{0}\right)\right)$, then $M\left(k_{C}^{\infty}\left(z_{0}\right)\right) \subset k_{M(C)}^{\infty}\left(M z_{0}\right)$.

(2) If $K_{M(C)}\left(M z_{0}\right) \subset M\left(K_{C}\left(z_{0}\right)\right)$, then $M\left(K_{C}^{\infty}\left(z_{0}\right)\right) \subset K_{M(C)}^{\infty}\left(M z_{0}\right)$.

ProOF. Let $y \in k_{C}^{\infty}\left(z_{0}\right)$, and call $z=M(y)$. Let $w \in k_{M(C)}\left(M z_{0}\right)$. By hypothesis, $w=M(v)$ for some $v \in k_{C}\left(z_{0}\right)$. Hence $z+w=M(y+v)$, and since $y+v \in k_{C}\left(z_{0}\right)$, we have $z+w \in M\left(k_{C}\left(z_{0}\right)\right) \subset k_{M(C)}\left(M z_{0}\right)$. Therefore $z \in k_{M(C)}^{\infty}\left(M z_{0}\right)$, and implication (1)is established. The proof of (2) is completely analogous to that of $(1)$.

Proposition 2.4. Let $C$ be a nonempty subset of $E, z_{0} \in \mathrm{cl} C$, and $M: E \rightarrow$ $E_{1}$ a continuous linear mapping satisfying the following condition:

$$
\begin{aligned}
& \text { Whenever }\left(w_{n}, t_{n}\right) \rightarrow\left(M z_{0}, 0^{+}\right) \text {such that } w_{n} \in M(C) \text { and } \\
& t_{n}^{-1}\left(w_{n}-M z_{0}\right) \text { converges, there exists } z_{n} \in C \text { with } w_{n}=M\left(z_{n}\right) \\
& \text { and } t_{n}^{-1}\left(z_{n}-z_{0}\right) \text { convergent. }
\end{aligned}
$$

Then for $A:=P, K^{\infty}$, and $k^{\infty}$,

$$
M\left(A_{C}\left(z_{0}\right)\right) \subset A_{M(C)}\left(M z_{0}\right) .
$$

ProOf. Let $v \in M\left(P_{C}\left(z_{0}\right)\right)$. Then $v=M(y)$ with $y \in P_{C}\left(z_{0}\right)$. Suppose $\left(w_{n}, t_{n}\right) \rightarrow\left(M z_{0}, 0^{+}\right)$such that $w_{n} \in M(C)$ and $t_{n}^{-1}\left(w_{n}-M z_{0}\right)$ converges. By (2.12), there exists $z_{n} \in C$ with $w_{n}=M\left(z_{n}\right)$ and $t_{n}^{-1}\left(z_{n}-z_{0}\right)$ convergent. There then exists $y_{n} \rightarrow y$ such that $z_{n}+t_{n} y_{n} \in C$. Hence $M y_{n} \rightarrow M y$ and $w_{n}+t_{n} M\left(y_{n}\right)=$ $M\left(z_{n}+t_{n} y_{n}\right) \in M(C)$. Therefore $v \in P_{M(C)}\left(M z_{0}\right)$ and (2.13) is established for $A:=P$. To prove (2.13) for $A:=k^{\infty}$, it suffices by Lemma 2.3 to show that $k_{M(C)}\left(M z_{0}\right) \subset M\left(k_{C}\left(z_{0}\right)\right)$. Let $v \in k_{M(C)}\left(M z_{0}\right)$ and $t_{n} \downarrow 0$. There exists $v_{n} \rightarrow v$ such that $w_{n}:=M z_{0}+t_{n} v_{n} \in M(C)$. Since $v_{n}=t_{n}^{-1}\left(w_{n}-M z_{0}\right)$ converges, there exists by $(2.12)$ a sequence $\left\{z_{n}\right\} \subset C$ such that $w_{n}=M\left(z_{n}\right)$ and $y_{n}:=t_{n}^{-1}\left(z_{n}-z_{0}\right)$ converges to some $y \in E$. Now $v_{n}=M\left(y_{n}\right)$, so $v=M(y)$, and since $x_{0}+t_{n} y_{n}=z_{n} \in C, y \in k_{C}\left(z_{0}\right)$. Thus $v \in M\left(k_{C}\left(z_{0}\right)\right)$. The proof for the $A:=K^{\infty}$ case is completely analogous to that for $k^{\infty}$.

We will make use of Proposition 2.4 in proving calculus formulae in $\S 5$.

3. The inversion theorem and tangent cone inclusions. A key ingredient in the proofs of the subdifferential calculus formulae we will present in $\S 4$ is a tangent cone inclusion derived by means of an "inversion theorem", a special case of $[\mathbf{4}$, Theorem 4.1] (see also $[\mathbf{1 3}, \mathbf{2}, \mathbf{1}]$ ).

We begin with some preliminary definitons.

DEFinition 3.1. Let $E$ and $E_{1}$ be Banach spaces. A function $G: E \rightarrow E_{1}$ is said to be strictly differentiable at $x_{0} \in E$ if there exists a continuous linear mapping $\nabla G\left(x_{0}\right): E \rightarrow E_{1}$ such that

$$
\lim _{\left(x, y^{\prime}, t\right) \rightarrow\left(x_{0}, y, 0^{+}\right)} t^{-1}\left[G\left(x+t y^{\prime}\right)-G(x)\right]=\nabla G\left(x_{0}\right) y
$$

for all $y \in E$. It is Hadamard differentiable at $x_{0}$ if for all $y \in E$,

$$
\lim _{\left(y^{\prime}, t\right) \rightarrow\left(y, 0^{+}\right)} t^{-1}\left[G\left(x_{0}+t y^{\prime}\right)-G\left(x_{0}\right)\right]=\nabla G\left(x_{0}\right) y .
$$


DEFinition 3.2 (CF. [27]). A nonempty $C \subset E$ is closed near $x_{0} \in \operatorname{cl} C$ if $N_{\varepsilon}\left(x_{0}\right) \cap C$ is closed for some $\varepsilon>0$. A function $f: E \rightarrow \overline{1 \mathbf{R}}$ which is finite at $x_{0}$ is said to be strictly l.s.c. at $x_{0}$ if epi $f$ is closed near $\left(x_{0}, f\left(x_{0}\right)\right)$.

DEFINITION 3.3 [3]. Let $C$ be a nonempty subset of $E$ that is closed near $x$.

(a) The set $C$ is said to be epi-Lipschitz-like at $x$ if there exist $\delta>0$, a convex set $\Omega$ with $\Omega^{0}$ weak-star locally compact, and $\lambda>0$ such that for all $t \in(0, \lambda)$, $C \cap N_{\delta}(x)+t \Omega \subset C$.

(b) Let $f: E \rightarrow \overline{1 \mathbf{R}}$ be strictly l.s.c. at $x$. The function $f$ is said to be Lipschitzlike at $x$ if epi $f$ is epi-Lipschitz-like at $(x, f(x))$.

Observe that if $E$ is finite-dimensional, any locally closed set is epi-Lipschitz-like, since $\Omega$ may be chosen to be $\{0\}$. An epi-Lipschitzian set in a normed space $E$ is epi-Lipschitz-like, since $\Omega$ may be chosen to be a neighborhood of some point. Thus strictly l.s.c. functions with finite-dimensional domains and epi-Lipschitzian functions with normed space domains are Lipschitz-like. Also, we note that products of epi-Lipschitz-like sets are epi-Lipschitz-like. This fact will be important in $\S 4$.

The inversion theorem of [4, Theorem 4.1] unifies the finite-dimensional and Banach space cases treated separately in [2]. We will use the following special case of this theorem.

THEOREM 3.4 [4]. Let $E$ and $E_{1}$ be Banach spaces, and let $G: E \rightarrow E_{1}$ be strictly differentiable on $N_{\lambda}\left(x_{0}\right)$ for some $\lambda>0$, where $x_{0} \in G^{-1}(0) \cap D$ and $D$ is epi-Lipschitz-like at $x_{0}$. Suppose

$$
\nabla G\left(x_{0}\right) T_{D}\left(x_{0}\right)=E_{1} .
$$

Then there exist $K>0$ and $\delta>0$ such that for each $x \in D \cap N_{\delta}\left(x_{0}\right)$, there exists $d \in D \cap G^{-1}(0)$ satisfying $\|x-d\| \leq K\|G(x)\|$.

Theorem 3.4 may be proved by means of Ekeland's variational principle [1]. Because of the presence of $T_{D}\left(x_{0}\right)$ in assumption (3.1), the hypotheses of all our main results will involve the Clarke tangent cone. We will later give an example (Remark 4.5(d)) which shows that $T$ cannot be replaced by $K, k, K^{\infty}, k^{\infty}$, or $P$ in (3.1). (See [2] for a similar example.) This seems to indicate that the Clarke tangent cone occupies a special position in the theory developed here.

We will now utilize Theorem 3.4 to prove a number of tangent cone inclusions.

THEOREM 3.5. Under the hypotheses of Theorem 3.4,

$$
A_{D}\left(x_{0}\right) \cap \nabla G\left(x_{0}\right)^{-1}(0) \subset A_{D \cap G^{-1}(0)}\left(x_{0}\right)
$$

for $A:=T, P, k^{\infty}, K^{\infty}, k$, and $K$.

ProOF. The cases $A=T, K$ are proven in [2, Theorem 4.1]. We include here the proof of the $A:=k^{\infty}$ case. Let $y \in k_{D}^{\infty}\left(x_{0}\right) \cap \nabla G\left(x_{0}\right)^{-1}(0)$, and suppose $z \in k_{D \cap G^{-1}(0)}\left(x_{0}\right)$. It suffices to show that $y+z \in k_{D \cap G^{-1}(0)}$. Since $k$ is isotone, $z \in k_{D}\left(x_{0}\right)$ and $z \in k_{G^{-1}(0)}\left(x_{0}\right) \subset \nabla G\left(x_{0}\right)^{-1}(0)$. So if $t_{n} \rightarrow 0^{+}$, there exists $w_{n} \rightarrow y+z$ such that $x_{0}+t_{n} w_{n} \in D$. Now since $G$ is strictly differentiable at $x_{0}$,

$$
t_{n}^{-1}\left(G\left(x_{0}+t_{n} w_{n}\right)-G\left(x_{0}\right)\right) \rightarrow \nabla G\left(x_{0}\right)(y+z)=0 .
$$

It follows, then, from Theorem 3.4 that there exists $d_{n} \in D \cap G^{-1}(0)$ such that $t_{n}^{-1}\left(d_{n}-x_{0}-t_{n} w_{n}\right) \rightarrow 0$ also. Let $v_{n}:=t_{n}^{-1}\left(d_{n}-x_{0}\right)$. Then $v_{n} \rightarrow y+z$ and 
$x_{0}+t_{n} v_{n}=d_{n} \in D \cap G^{-1}(0)$. Therefore $y+z \in k_{D \cap G^{-1}(0)}\left(x_{0}\right)$, and the proof is complete. The proofs for the cases $A:=k, K^{\infty}$, and $P$ are quite similar to this one.

4. Calculus for directional derivatives and subgradients. We begin this section by reviewing the now familiar idea of associating directional derivatives and subgradients with tangent cones $[\mathbf{1 2}, \mathbf{2 6}]$.

DEFinition 4.1. Let $f: E \rightarrow \overline{1 \mathrm{R}}$ be finite at $x_{0} \in E$. For a tangent cone $A$ the $A$ directional derivative of $f$ at $x_{0}$ in the direction $y$ is defined by

$$
f^{A}\left(x_{0} ; y\right):=\inf \left\{r \mid(y, r) \in A_{f}\left(x_{0}\right)\right\} .
$$

The $A$ subgradient of $f$ at $x_{0}$ is the set

$$
\partial^{A} f\left(x_{0}\right):=\left\{x^{\prime} \in E^{\prime} \mid\left\langle x^{\prime}, y\right\rangle \leq f^{A}\left(x_{0} ; y\right) \text { for all } y \in E\right\} .
$$

Definition 4.1 is designed precisely so that

$$
\text { epi } f^{A}\left(x_{0} ; \cdot\right)=A_{f}\left(x_{0}\right)
$$

if $A$ is a closed tangent cone (in particular, for $A:=K, k, K^{\infty}, k^{\infty}, P$ and $T$ ).

It is well known that if $G: E \rightarrow \overline{\mathbf{1 R}}$ is strictly differentiable at $x_{0}$,

$$
\partial^{A} G\left(x_{0}\right)=\left\{\nabla G\left(x_{0}\right)\right\}
$$

for any $A$ satisfying (1.2). If $G$ is merely Hadamard differentiable, (4.4) remains true for $A$ such that $P \subset A \subset K[\mathbf{2 2}]$. This is one advantage of $P, k^{\infty}, K^{\infty}, k$, and $K$ over $T$.

Equation (4.3) and the tangent cone properties discussed in $\S \S 2$ and 3 can be combined to prove calculus formulae for $f^{A}$, which will in turn produce corresponding formulae for $\partial^{A} f$ if $A$ is convex. This was demonstrated in detail for $A:=T, k$, and $K$ in [33]. Roughly speaking, if a closed tangent cone $A$ satisfies (2.5), (2.6) for the appropriate $M, C$, and $z_{0}$ and (3.2) (under assumption (3.1)), then $f^{A}$ will have an extensive calculus including rules for sums and pointwise maxima of functions, products of positive-valued functions, and compositions $f=g \circ F$ where either $g: 1 \mathbf{R}^{m} \rightarrow \overline{1 \mathbf{R}}, F: E \rightarrow 1 \mathbf{R}^{m}$, and $g$ is nondecreasing or $g: E_{1} \rightarrow \overline{1 \mathrm{R}}, F: E \rightarrow E_{1}$, and $F$ is strictly differentiable. In other words, an "algorithm" for generating a calculus for $f^{A}$ consists simply of checking $(2.5),(2.6)$, and Theorem 3.4 for $A$. We establish the details of this procedure in this section and apply it to $k^{\infty}$ and $P$ in $\S 5$. In this section, we will assume that $A$ and $A^{\prime}$ are closed tangent cones which satisfy $T \subset A^{\prime} \subset A \subset K$ and

$$
A_{C}\left(x_{0}\right) \times A_{D}^{\prime}\left(y_{0}\right) \subset A_{C \times D}\left(x_{0}, y_{0}\right)
$$

in general. We assume in addition that (3.2) is true for $A$ under condition (3.1). For example, $A=A^{\prime}=T, k, k^{\infty}, P$, as well as $A=K, A^{\prime}=k$ fit this description.

We now consider the first of two chain rule formulations. Here, as in [25], we adopt the convention that $\infty-\infty=\infty$.

THEOREM 4.2. Let $E$ and $E_{1}$ be Banach spaces and $F: E \rightarrow E_{1}$ strictly differentiable on $N_{\delta}\left(x_{0}\right)$ for some $\delta>0$. Let $f_{1}: E \rightarrow \overline{1 \mathbf{R}}$ be finite and Lipschitz-like at $x_{0}$ and $f_{2}: E_{1} \rightarrow \overline{\mathbf{1 R}}$ finite and Lipschitz-like at $F\left(x_{0}\right)$. Suppose $A$ and $A^{\prime}$ are 
closed tangent cones satisfying $T \subset A^{\prime} \subset A \subset K$ and (4.5), with (3.2) valid for $A$ under conditon (3.1). Assume

$$
\nabla F\left(x_{0}\right) \operatorname{dom} f_{1}^{T}\left(x_{0}, \cdot\right)-\operatorname{dom} f_{2}^{T}\left(F\left(x_{0}\right) ; \cdot\right)=E_{1} .
$$

Define $M: E \times 1 \mathbf{R} \times E_{1} \times 1 \mathbf{R} \rightarrow E \times 1 \mathbf{R}$ by $M(x, y, z, r)=(x, y+r)$ and $G: E \times 1 \mathbf{R} \times E_{1} \times 1 \mathbf{R} \rightarrow E$, by $G(x, y, z, r)=F(x)-z$. Assume that (2.6) holds for $M$ as above, $z_{0}:=\left(x_{0}, f_{1}\left(x_{0}\right), F\left(x_{0}\right), f_{2}\left(F\left(x_{0}\right)\right)\right)$, and $C:=\left(\right.$ epi $f_{1} \times$ epi $\left.f_{2}\right) \cap G^{-1}(0)$. Then for all $y \in E$,

$$
\left(f_{1}+f_{2} \circ F\right)^{A}\left(x_{0} ; y\right) \leq f_{1}^{A}\left(x_{0} ; y\right)+f_{2}^{A^{\prime}}\left(F\left(x_{0}\right) ; \nabla F\left(x_{0}\right) y\right) .
$$

Moreover, if $A$ and $A^{\prime}$ are convex, then

$$
\partial^{A}\left(f_{1}+f_{2} \circ F\right)\left(x_{0}\right) \subset \partial^{A} f_{1}\left(x_{0}\right)+\nabla F\left(x_{0}\right)^{*} \partial^{A^{\prime}} f_{2}\left(F\left(x_{0}\right)\right) .
$$

Equality holds in (4.8) if $f_{1}^{A}\left(x_{0} ; \cdot\right)=f_{1}^{K}\left(x_{0} ; \cdot\right)$ and $f_{2}^{A^{\prime}}\left(F\left(x_{0}\right) ; \nabla F\left(x_{0}\right)(\cdot)\right)=$ $f_{2}^{K}\left(F\left(x_{0}\right) ; \nabla F\left(x_{0}\right)(\cdot)\right)$. Equality holds in $(4.7)$ if in addition $f_{1}^{K}\left(x_{0} ; \cdot\right)$ and $f_{2}^{K}\left(F\left(x_{0}\right) ; \cdot\right)$ are proper.

Proof. Call $f:=f_{1}+f_{2} \circ F$. Then

$$
\text { epi } \begin{aligned}
f=\left\{\left(x_{1}, r_{1}+r_{2}\right) \mid\right. & f_{1}\left(x_{1}\right) \leq r_{1}, f_{2}\left(x_{2}\right) \leq r_{2}, \\
& \left.F\left(x_{1}\right)-x_{2}=0 \text { for some } x_{2} \in E_{1}\right\} .
\end{aligned}
$$

Define $D:=\operatorname{epi} f_{1} \times$ epi $f_{2}$. Note that $D$ is epi-Lipschitz-like at $z_{0}$. By our definitions, $M\left(D \cap G^{-1}(0)\right)=$ epi $f$, and so

$$
\text { epi } f^{A}\left(x_{0} ; \cdot\right)=A_{M\left(D \cap G^{-1}(0)\right)}\left(x_{0}, f\left(x_{0}\right)\right) \supset M\left(A_{D \cap G^{-1}(0)}\left(z_{0}\right)\right)
$$

by hypotheses. Next observe that (4.6) and (2.8) ensure that $\nabla G\left(z_{0}\right) T_{D}\left(z_{0}\right)=E_{1}$. Since $A$ satisfies (3.2) under this condition,

$$
A_{D \cap G^{-1}(0)}\left(z_{0}\right) \supset A_{D}\left(z_{0}\right) \cap \nabla G\left(z_{0}\right)^{-1}(0) .
$$

Thus

$$
\begin{aligned}
M\left(A_{D \cap G^{-1}(0)}\left(z_{0}\right)\right) & \supset M\left(A_{D}\left(z_{0}\right) \cap \nabla G\left(z_{0}\right)^{-1}(0)\right) \\
& \supset M\left(\left(\operatorname{epi} f_{1}^{A}\left(z_{0}: \cdot\right) \times \operatorname{epi} f_{2}^{A^{\prime}}\left(F\left(x_{0}\right) ; \cdot\right)\right) \cap \nabla G\left(z_{0}\right)^{-1}(0)\right)
\end{aligned}
$$

by $(4.5)$

$$
\begin{aligned}
& =M\left(\left\{\left(h_{1}, r_{1}, h_{2}, r_{2}\right) \mid f_{1}^{A}\left(x_{0} ; h_{1}\right) \leq r_{1}, f_{2}^{A^{\prime}}\left(F\left(x_{0}\right) ; h_{2}\right) \leq r_{2}, \nabla F\left(x_{0}\right) h_{1}=h_{2}\right\}\right) \\
& =\left\{\left(h, r_{1}+r_{2}\right) \mid f_{1}^{A}\left(x_{0} ; h\right) \leq r_{1}, f_{2}^{A^{\prime}}\left(F\left(x_{0}\right) ; \nabla F\left(x_{0}\right) h\right) \leq r_{2}\right\} \\
& =\operatorname{epi}\left[f_{1}^{A}\left(x_{0} ; \cdot\right)+f_{2}^{A^{\prime}}\left(F\left(x_{0}\right) ; \nabla F\left(x_{0}\right)(\cdot)\right)\right] .
\end{aligned}
$$

Therefore (4.7) holds. If $A$ and $A^{\prime}$ are convex, set $p_{1}(\cdot):=f_{1}^{A}\left(x_{0} ; \cdot\right)$ and $p_{2}(\cdot):=$ $f_{2}^{A^{\prime}}\left(F\left(x_{0}\right) ; \cdot\right)$. If either $p_{1}(0)$ or $p_{2}(0)$ is $-\infty,(4.7)$ shows that both sides of $(4.8)$ wil then be empty. We may assume, then, that $p_{1}(0)=p_{2}(0)=0$. Then

$$
\begin{aligned}
\partial^{A} f\left(x_{0}\right) & =\left\{z \in E^{\prime} \mid\left(f_{1}+f_{2} \circ F\right)^{A}\left(x_{0} ; y\right) \geq\langle z, y\rangle \text { for all } y \in E\right\} \\
& =\left\{z \mid p_{1}(y)+p_{2}\left(\nabla F\left(x_{0}\right) y\right) \geq\langle z, y\rangle \text { for all } y \in E\right\} \\
& =\partial\left(p_{1}+p_{2} \circ \nabla F\left(x_{0}\right)\right)(0) .
\end{aligned}
$$

Since $T \subset A^{\prime} \subset A$ and (4.6) holds, it follows that $\nabla F\left(x_{0}\right) \operatorname{dom} p_{1}-\operatorname{dom} p_{2}=E_{1}$. Now $p_{1}$ and $p_{2}$ are proper and sublinear, so we have by the subdifferential calculus 
of sublinear functions (see $[\mathbf{1 7}, \mathbf{1 . 2 . 5}]$, or in finite dimensions [24, Theorems 23.8, 23.9]) that

$$
\begin{aligned}
\partial\left(p_{1}+p_{2} \circ \nabla F\left(x_{0}\right)\right)(0) & =\partial p_{1}(0)+\nabla F\left(x_{0}\right)^{*} \partial p_{2}(0) \\
& =\partial^{A} f_{1}\left(x_{0}\right)+\nabla F\left(x_{0}\right)^{*} \partial^{A^{\prime}} f_{2}\left(F\left(x_{0}\right)\right)
\end{aligned}
$$

and so (4.8) holds. Finally, if $f_{1}^{A}\left(x_{0} ; \cdot\right)=f_{1}^{K}\left(x_{0} ; \cdot\right)$ and $f_{2}^{A^{\prime}}\left(F\left(x_{0}\right) ; \nabla F\left(x_{0}\right)(\cdot)\right)=$ $f_{2}^{K}\left(F\left(x_{0}\right) ; \nabla F\left(x_{0}\right)(\cdot)\right)$,

$$
\begin{aligned}
\left(f_{1}+f_{2} \circ F\right)^{A}\left(x_{0} ; \cdot\right) & \geq\left(f_{1}+f_{2} \circ F\right)^{K}\left(x_{0} ; \cdot\right) \\
& \geq f_{1}^{K}\left(x_{0} ; \cdot\right)+f_{2}^{K}\left(F\left(x_{0}\right) ; \nabla F\left(x_{0}\right)(\cdot)\right)
\end{aligned}
$$

(if $f_{1}^{K}\left(x_{0} ; \cdot\right)$ and $f_{2}^{K}\left(F\left(x_{0}\right) ; \cdot\right)$ are proper)

$$
=f_{1}^{A}\left(x_{0} ; \cdot\right)+f_{2}^{A^{\prime}}\left(F\left(x_{0}\right) ; \nabla F\left(x_{0}\right)(\cdot)\right) \text {. }
$$

Hence equality holds in (4.7) and (4.8) under the stated assumptions.

REMARK 4.3. (a) Condition (4.6) is satisfied by quite general classes of functions. For example, this conditon holds in any of the following cases:

(i) $\nabla F\left(x_{0}\right)$ is surjective and $f_{1}$ is locally Lipschitzian near $x_{0}$.

(ii) $f_{2}$ is locally Lipschitzian near $F\left(x_{0}\right)$.

(iii) $f_{2}$ is directionally Lipschitzian at $F\left(x_{0}\right)$ and

$$
\nabla F\left(x_{0}\right) \operatorname{dom} f_{1}^{T}\left(x_{0} ; \cdot\right) \cap \operatorname{int} \operatorname{dom} f_{2}^{T}\left(F\left(x_{0}\right) ; \cdot\right) \neq \varnothing .
$$

For further discussion, see [33].

(b) If $A \subset k$, the conditions for equality in (4.7) and (4.8) can be sharpened [33]. In this case, either $f_{1}^{A}\left(x_{0} ; \cdot\right)=f_{1}^{k}\left(x_{0} ; \cdot\right)$ and

$$
f_{2}^{A^{\prime}}\left(F\left(x_{0}\right) ; \nabla F\left(x_{0}\right)(\cdot)\right)=f_{2}^{K}\left(F\left(x_{0}\right) ; \nabla F\left(x_{0}\right)(\cdot)\right)
$$

or

$$
f_{1}^{A}\left(x_{0} ; \cdot\right)=f_{1}^{K}\left(x_{0} ; \cdot\right) \quad \text { and } \quad f_{2}^{A^{\prime}}\left(F\left(x_{0}\right) ; \nabla F\left(x_{0}\right)(\cdot)\right)=f_{2}^{k}\left(F\left(x_{0}\right) ; \nabla F\left(x_{0}\right)(\cdot)\right)
$$

will guarantee equality in (4.8).

(c) The roles of $A$ and $A^{\prime}$ may be reversed in the right-hand side of (4.7) and (4.8).

The special cases of Theorem 4.2 where $A:=K, A^{\prime}:=k, A=A^{\prime}:=k$, and $A=A^{\prime}:=T$ are discussed in detail in [33]. A number of corollaries of Theorem 4.2 , analogous to those listed for the $A=A^{\prime}:=T$ case in $[33, \S 3]$ can be proven. We will concentrate our attention here on just one of them, after making some preliminary definitions.

DEFINITON 4.4. Let $C$ be a nonempty subset of $E$. Define

$$
\Delta^{n} C:=\left\{\left(x_{1}, \ldots, x_{n}\right) \in C \mid x_{1}=x_{2}=\cdots=x_{n}\right\} .
$$

DEFINITION 4.5. Let $C_{i} \subset E, i=1, \ldots, n$, be nonempty convex sets. These sets are said to be in strong general position [36] if

$$
0 \in \operatorname{int}\left[\Delta^{n-1} C_{1}-\prod_{j=2}^{n} C_{j}\right] .
$$


It is shown in [36] that (4.9) is equivalent to

$$
0 \in \operatorname{int}\left[\Delta^{n} E-\prod_{j=1}^{n} C_{j}\right] \text {. }
$$

If the sets $C_{i}, i=1, \ldots, n$, are cones, then (4.9) can be written

$$
\Delta^{n-1} C_{1}-\prod_{j=2}^{n} C_{j}=E^{n-1} .
$$

Proposition 4.6 (CF. [33, Proposition 3.10, Corollary 6.15]). Let $A$ and $A^{\prime}$ be tangent cones as in Theorem 4.2, and let $D_{i} \subset E, i=1, \ldots, n$, be epiLipschitz-like at $y_{0} \in \bigcap_{i=1}^{n} D_{i}$. Assume $T_{D_{i}}\left(y_{0}\right), i=1, \ldots, n$, are in strong general position. Then

$$
A_{D_{1} \cap \cdots \cap D_{n}}\left(y_{0}\right) \supset A_{D_{1}}\left(y_{0}\right) \cap\left(\bigcap_{i=2}^{n} A_{D_{i}}^{\prime}\left(y_{0}\right)\right) .
$$

Moreover, if $A$ and $A^{\prime}$ are convex, then

$$
\left(A_{D_{1} \cap \cdots \cap D_{n}}\left(y_{0}\right)\right)^{0} \subset\left(A_{D_{1}}\left(y_{0}\right)\right)^{0}+\sum_{i=2}^{n}\left(A_{D_{i}}^{\prime}\left(y_{0}\right)\right)^{0}:
$$

Equality holds in (4.12) and (4.13) if

$$
A_{D_{1}}\left(y_{0}\right)=K_{D_{1}}\left(y_{0}\right) \quad \text { and } \quad A_{D_{i}}^{\prime}\left(y_{0}\right)=K_{D_{i}}\left(y_{0}\right), \quad i=2, \ldots, n .
$$

ProOF. Define $f_{1}:=i_{D_{1} \times \cdots \times D_{n}}$ and $f_{2}:=i_{\{0\}}$, where $\{0\}$ denotes the origin in $E^{n-1}$. Define $F: E^{n} \rightarrow E^{n-1}$ by $F\left(x_{1}, \ldots, x_{n}\right):=\left(x_{1}-x_{2}, \ldots, x_{1}-x_{n}\right)$. Observe that the relationships $i_{C}^{A}\left(x^{\prime} ; \cdot\right)=i_{A_{C}(x)}(\cdot)$ and $\partial^{A} i_{C}(x)=\left(A_{C}(x)\right)^{0}$ hold for any nonempty $C \subset E^{n}$ and $x \in C$, and for $A^{\prime}$ as well as $A$. Apply Theorem 4.2 with $x_{0}:=\left(y_{0}, \ldots, y_{0}\right)$. Since $T$ satisfies (2.5), the strong general position assumption guarantees that (4.6) holds. Then (4.12) and (4.13) follow from (4.7) and (4.8), respectively, since $A$ and $A^{\prime}$ satisfy (4.5). Since $i_{C}^{K}\left(x_{0} ; \cdot\right)$ is proper for any nonempty $C$ and $x \in C$, the stated conditons for equality follow from those in Theorem 4.2.

REMARK 4.7. (a) An application of Proposition 4.6 with $D_{i}:=\operatorname{epi} f_{i}$ will give a calculus rule for $f^{A}$ and $\partial^{A} f$ where $f(x):=\max _{1 \leq i \leq n} f_{i}(x)$ (see [33, Proposition $3.14])$.

(b) If $A \subset k$, then the conditions for equality in Proposition 4.6 can be sharpened to $A_{D_{1}}\left(y_{0}\right)=k_{D_{1}}\left(y_{0}\right)$ and $A_{D_{i}}^{\prime}\left(y_{0}\right)=k_{D_{i}}\left(y_{0}\right), i=2, \ldots, n$.

Under these conditions,

$$
A_{D_{i} \cap \cdots \cap D_{n}}\left(y_{0}\right) \subset k_{D_{1} \cap \cdots \cap D_{n}}\left(y_{0}\right) \subset \bigcap_{i=1}^{n} k_{D_{i}}\left(y_{0}\right)
$$

since $k$ is isotone.

(c) Inclusion (4.12) for $A=A^{\prime}=T$ was established by Watkins in [35] to show that the Clarke tangent cone satisfies the intersection principle of Martin, Gardner, and Watkins [18]. As a result, a Dubovitskii-Milyutin approach may be used to 
prove quite general Fritz John type Lagrange multiplier rules involving $\partial^{T} f[\mathbf{3 5}$, 33].

(d) We will see in $\S 5$ that $A=A^{\prime}:=P$ and $A=A^{\prime}:=k^{\infty}$ can be used in Theorem 4.2 and Proposition 4.6. However, in the hypotheses of these results, $T$ cannot be replaced by $k^{\infty}$ or $P$, as we now demonstrate. Define

$$
D_{1}:=\left\{(x, y) \in 1 \mathbf{R}_{+}^{2} \mid x+y=1 / n, n \text { odd }\right\} \cup\{0,0\}
$$

and

$$
D_{2}:=\left\{(x, y) \in 1 \mathbf{R}_{+}^{2} \mid x+y=1 / n, n \text { even }\right\} \cup\{(0,0)\} .
$$

Then $k_{D_{i}}(0,0)=1 \mathbf{R}_{+}^{2}, i=1,2$, so that $k_{D_{i}}^{\infty}(0,0)=P_{D_{i}}(0,0)=1 \mathbf{R}_{+}^{2}$. In this example, $A_{D_{i}}(0,0), i=1,2$, are in strong general position (i.e., $A_{D_{1}}(0,0)-A_{D_{2}}(0,0)=$ $\left.1 \mathbf{R}^{2}\right)$ for $A:=k^{\infty}, P$. Inclusion (4.12) with $n=2$ does not hold for $A=A^{\prime}:=P$ or $A=A^{\prime}:=k^{\infty}$, though, since $D_{1} \cap D_{2}=\{(0,0)\}$. This example also demonstrates that $T$ cannot be replaced by $k^{\infty}$ or $P$ in (3.1), and that $P$ and $k^{\infty}$ do not satisfy the intersection principle mentioned in (c).

DEFINITION 4.8. Let $x=\left(x_{1}, \ldots, x_{n}\right)$ and $\left(y_{1}, \ldots, y_{n}\right)$ be elements of $1 \mathbf{R}^{n}$. We say $x \leq y$ if $x_{i} \leq y_{i}$ for each $i$. The function $F: 1 \mathbf{R}^{n} \rightarrow \overline{1 \mathbf{R}}$ is isotone on $B \subset 1 \mathbf{R}^{n}$ if $F(x) \leq F(y)$ whenever $x, y \in B$ and $x \leq y$.

We now establish a chain rule for compositions of the form $F \circ f$, where $f:=$ $\left(f_{1}, \ldots, f_{n}\right)$ each $f_{i}: E \rightarrow \overline{\mathbf{1}}$ is finite and Lipschitz-like at $x_{0}$, and $F: 1 \mathbf{R}^{n} \rightarrow \overline{\mathbf{1}}$ is finite at $f\left(x_{0}\right)$, l.s.c., and isotone on $N_{\delta}\left(f\left(x_{0}\right)\right) \cup B$ for some $\delta>0$, with

$$
B:=\left\{y \in 1 \mathbf{R}^{n} \mid f(x) \leq y \text { for some } x \in E\right\} .
$$

In such a composition, we define

$$
(F \circ f)(x)=\inf \left\{F(y) \mid f(x) \leq y, y \in 1 \mathbf{R}^{n}\right\},
$$

and set $F(f(x))=+\infty$ if some $f_{i}(x)=+\infty$. The proof of this chain rule will depend on another special case of (2.6).

THEOREM 4.9. Let $F$ and $f$ be defined as in the preceding paragraph. Suppose $A$ and $A^{\prime}$ are closed tangent cones satisfying $T \subset A^{\prime} \subset A \subset K$ and (4.5), with (3.2) valid for $A$ under condition (3.1). Assume that $F^{A^{\prime}}\left(f\left(x_{0}\right) ; \cdot\right)$ is isotone on $1 \mathbf{R}^{n}$, that $f_{1}^{A}\left(x_{0} ; \cdot\right)$ and $f_{i}^{A^{\prime}}\left(x_{0} ; \cdot\right), i=2, \ldots, n$, are proper, and that

$$
\left(\Delta^{n} E \times \operatorname{dom} F^{T}\left(f\left(x_{0}\right) ; \cdot\right)\right)-S=E^{n} \times 1 \mathbf{R}^{n},
$$

where

$$
S:=\left\{\left(y_{1}, \ldots, y_{n}, r_{1}, \ldots, r_{n}\right) \mid\left(y_{i}, r_{i}\right) \in \operatorname{epi} f_{i}^{T}\left(x_{0} ; \cdot\right), i=1, \ldots, n\right\} .
$$

Define $M:(E \times 1 \mathbf{R})^{n} \times 1 \mathbf{R}^{n+1} \rightarrow E \times 1 \mathbf{R}$ by

$$
M\left(x_{1}, y_{1}, \ldots, x_{n}, y_{n}, z_{1}, \ldots, z_{n}, r\right)=\left(x_{1}, r\right)
$$

and $G:(E \times 1 \mathbf{R})^{n} \times 1 \mathbf{R}^{n+1} \rightarrow E^{n-1} \times 1 \mathbf{R}^{n}$ by

$$
G\left(x_{1}, y_{1}, \ldots, x_{n}, y_{n}, z_{1}, \ldots, z_{n}, r\right)=\left(x_{1}-x_{2}, \ldots, x_{1}-x_{n}, y_{1}-z_{1}, \ldots, y_{n}-z_{n}\right) .
$$

Assume that (2.6) holds for this $M$ and $C:=\left(\prod_{i=1}^{n}\right.$ epi $f_{i} \times$ epi $\left.F\right) \cap G^{-1}(0), z_{0}:=$ $\left(x_{0}, f_{1}\left(x_{0}\right), \ldots, x_{0}, f_{n}\left(x_{0}\right), f_{1}\left(x_{0}\right), \ldots, f_{n}\left(x_{0}\right), F\left(f\left(x_{0}\right)\right)\right)$. Then for all $y \in E$,

$$
(F \circ f)^{A}\left(x_{0} ; y\right) \leq F^{A^{\prime}}\left(f\left(x_{0}\right) ; f_{1}^{A}\left(x_{0}, y\right), f_{2}^{A^{\prime}}\left(x_{0} ; y\right), \ldots, f_{n}^{A^{\prime}}\left(x_{0} ; y\right)\right) .
$$


Moreover, if $A$ and $A^{\prime}$ are convex, then

$$
\begin{aligned}
\partial^{A}(F \circ f) \subset\left\{\lambda \cdot\left(\partial^{A} f_{1}\left(x_{0}\right), \partial^{A^{\prime}} f_{2}\left(x_{0}\right), \ldots, \partial^{A^{\prime}} f_{n}\left(x_{0}\right)\right) \mid \lambda \geq 0^{+}\right. \\
\left.\lambda \in \partial k^{A^{\prime}} F\left(f\left(x_{0}\right)\right)\right\} .
\end{aligned}
$$

Equality holds in (4.16) if $F^{A^{\prime}}\left(f\left(x_{0}\right) ; \cdot\right)=F^{K}\left(f\left(x_{0}\right) ; \cdot\right), f_{1}^{A}\left(x_{0} ; \cdot\right)=f_{1}^{K}\left(x_{0} ; \cdot\right)$, and $f_{i}^{A^{\prime}}\left(x_{0} ; \cdot\right)=f_{i}^{K}\left(x_{0} ; \cdot\right), i=2, \ldots, n$. Equality holds in (4.15) if in addition $F^{K}\left(f\left(x_{0}\right) ; \cdot\right)$ is proper.

Proof. Call $h:=F \circ f$. Since $F$ is isotone on $B$,

epi $h=\left\{(x, r) \mid \exists\left(y_{1}, \ldots, y_{n}\right) \in 1 \mathbf{R}^{n}\right.$ with $F\left(y_{1}, \ldots, y_{n}\right) \leq r$

$$
\left.f_{i}(x) \leq y_{i}, i=1, \ldots, n\right\} \text {. }
$$

Define $D:=$ epi $f_{1} \times \cdots \times$ epi $f_{n} \times$ epi $F$, so that $C=D \cap G^{-1}(0)$. Then epi $h=$ $M(C)$, and we have

$$
\begin{aligned}
\operatorname{epi} h^{A}\left(x_{0} ; \cdot\right) & =A_{M(C)}\left(x_{0}, h\left(x_{0}\right)\right) \\
& \supset M\left(A_{C}\left(z_{0}\right)\right) \quad \text { by hypothesis } \\
& \supset M\left(A_{D}\left(z_{0}\right) \cap \nabla G\left(z_{0}\right)^{-1}(0)\right),
\end{aligned}
$$

since (4.14) ensures that (3.1) is satisfied

$$
\begin{aligned}
\supset\{(x, r) \mid & \exists y \in 1 \mathbf{R}^{n} \text { with } f_{1}^{A}\left(x_{0} ; x\right) \leq y_{1}, \\
& \left.f_{1}^{A^{\prime}}\left(x_{0} ; x\right) \leq y_{i}, i=2, \ldots, n, F^{A^{\prime}}\left(f\left(x_{0}\right) ; y\right) \leq r\right\} \quad \text { by }(4.5) \\
=\operatorname{epi} F^{A^{\prime}}\left(f\left(x_{0}\right) ; f_{1}^{A}\left(x_{0} ; \cdot\right), f_{2}^{A^{\prime}}\left(x_{0} ; \cdot\right), \ldots, f_{n}^{A^{\prime}}\left(x_{0} ; \cdot\right)\right) & \text { since } F^{A^{\prime}}\left(f\left(x_{0}\right) ; \cdot\right) \text { is isotone. }
\end{aligned}
$$

Thus (4.15) holds. Now suppose $A$ and $A^{\prime}$ are convex. If $F^{A^{\prime}}\left(f\left(x_{0}\right) ; 0\right)=-\infty$, (4.15) shows that both sides of (4.16) will be empty. We may assume, then, that $F^{A^{\prime}}\left(f\left(x_{0}\right) ; \cdot\right)$ is proper. Condition (4.14) implies $\operatorname{dom} f_{1}^{A}\left(x_{0} ; \cdot\right)$ and $\operatorname{dom} f_{i}^{A^{\prime}}\left(x_{0} ; \cdot\right)$, $i=2, \ldots, n$, are in strong general position, and that

$$
\begin{array}{r}
\operatorname{int} \operatorname{dom} F^{A^{\prime}}\left(f\left(x_{0}\right) ; \cdot\right) \cap\left\{\left(r_{1}, \ldots, r_{n}\right) \mid f_{1}^{A}\left(x_{0} ; y\right) \leq r_{1}, f_{i}^{A^{\prime}}\left(x_{0} ; y\right) \leq r_{i},\right. \\
i=2, \ldots, n, \text { for some } y \in E\} \neq \varnothing
\end{array}
$$

(see [33, Remark 2.11]). By the corresponding result from the subdifferential calculus for sublinear functions (the approriate analogue of [33, Theorem 2.10]),

$$
\begin{aligned}
\partial(F \circ f)\left(x_{0}\right) & =\left\{z \mid(F \circ f)^{A}\left(x_{0} ; y\right) \geq\langle z, y\rangle \forall y \in E\right\} \\
& \subset\left\{z \mid F^{A^{\prime}}\left(f\left(x_{0}\right) ; f_{1}^{A}\left(x_{0} ; y\right), f_{2}^{A^{\prime}}\left(x_{0} ; y\right), \ldots, f_{n}^{A^{\prime}}\left(x_{0}, y\right)\right)\right. \\
& \quad \geq\langle z, y\rangle \forall y \in E\} \\
& =\partial\left(\left(F^{A^{\prime}}\left(f\left(x_{0}\right) ; \cdot\right)\right) \circ\left(f_{1}^{A}\left(x_{0} ; \cdot\right), f_{2}^{A^{\prime}}\left(x_{0} ; \cdot\right), \ldots, f_{n}^{A^{\prime}}\left(x_{0} ; \cdot\right)\right)\right)(0) \\
& \left\{\lambda \cdot\left(\partial f_{1}^{A}\left(x_{0} ; \cdot\right)(0), \partial f_{2}^{A^{\prime}}\left(x_{0} ; \cdot\right)(0), \ldots, \partial f_{n}^{A^{\prime}}\left(x_{0} ;\right)(0)\right) \mid\right. \\
\left.\lambda \geq 0^{+}, \lambda \in \partial F^{A^{\prime}}\left(f\left(x_{0}\right) ; \cdot\right)(0)\right\} & \\
= & \left\{\lambda \cdot\left(\partial^{A} f_{1}\left(x_{0}\right), \partial^{A^{\prime}} f_{2}\left(x_{0}\right), \ldots, \partial^{A^{\prime}} f_{n}\left(x_{0}\right)\right) \mid\right. \\
\lambda & \left.\lambda \geq 0^{+}, \lambda \in \partial^{A^{\prime}} F\left(f\left(x_{0}\right)\right)\right\} .
\end{aligned}
$$


Finally, assume that the stated conditions for equality hold. Then for all $y \in E$,

$$
\begin{aligned}
(F \circ f)^{A}\left(x_{0} ; y\right) \geq & (F \circ f)^{K}\left(x_{0} ; y\right) \\
\geq & F^{K}\left(f\left(x_{0}\right) ; f_{1}^{K}\left(x_{0} ; y\right), \ldots, f_{n}^{K}\left(x_{0} ; y\right)\right) \\
& \quad \text { by }[\mathbf{3 3}, \text { Proposition 6.1] } \\
& =F^{A^{\prime}}\left(f\left(x_{0}\right) ; f_{1}^{A}\left(x_{0} ; y\right), f_{2}^{A^{\prime}}\left(x_{0} ; y\right), \ldots, f_{n}^{A^{\prime}}\left(x_{0} ; y\right)\right) .
\end{aligned}
$$

Therefore equality holds in (4.15) and (4.16).

The special cases of Theorem 4.9 where $A=A^{\prime}=T, A=A^{\prime}=k$, and $A=$ $K, A^{\prime}=k$ are covered in [33]. Corollaries of Theorem 4.9 include formulae for directional derivatives and subgradients of sums and pointwise maxima of functions, as well as product and quotient rules for positive-valued functions. Proposition 4.6 can be rederived via Theorem 4.9 .

5. Calculus for $P, k^{\infty}$, and $K^{\infty}$. In this section, we establish the special cases of Theorems 4.2 and 4.9 involving $A=A^{\prime}:=P$ and $A=A^{\prime}:=k^{\infty}$. We already have much relevant information about these tangent cones from Proposition 2.2 and Theorem 3.5; all that remains to be checked is inclusion (2.6) for the appropriate choices of $M, C$, and $z_{0}$. The case $A:=K^{\infty}$ is less satisfactory, since as we saw in $\S 2$ there is no $A^{\prime}$ to pair with $A:=K^{\infty}$ which will satisfy (4.5). Nevertheless, some results can be derived from known formulae for the $A:=K, A^{\prime}:=k$ case.

We now present the $A:=P, k^{\infty}$ cases of Theorem 4.2. In the proofs, we use the fact that

$$
f^{K}\left(x_{0} ; y\right)=\liminf _{\left(y^{\prime}, t\right) \rightarrow\left(y, 0^{+}\right)} t^{-1}\left[f\left(x_{0}+t y^{\prime}\right)-f\left(x_{0}\right)\right] .
$$

THEOREM 5.1. Let $F: E \rightarrow E_{1}$ be strictly differentiable on some $N_{\delta}\left(x_{0}\right), f_{1}$ : $E \rightarrow \overline{1 \mathbf{R}}$ finite and Lipschitz-like at $x_{0}$, and $f_{2}: E_{1} \rightarrow \overline{\mathbf{R}}$ finite and Lipschitzlike at $F\left(x_{0}\right)$. Assume that (4.6) holds, and that $f_{1}^{K}\left(x_{0} ; \cdot\right)$ and $\left(f_{2} \circ F\right)^{K}\left(x_{0} ; \cdot\right)$ are proper. Then for all $y \in E$,

$$
\begin{aligned}
& \left(f_{1}+f_{2} \circ F\right)^{k \infty}\left(x_{0} ; y\right) \leq f_{1}^{k \infty}\left(x_{0} ; y\right)+f_{2}^{k \infty}\left(F\left(x_{0}\right) ; \nabla F\left(x_{0}\right) y .\right. \\
& \left(f_{1}+f_{2} \circ F\right)^{P}\left(x_{0} ; y\right) \leq f_{1}^{P}\left(x_{0} ; y\right)+f_{2}^{P}\left(F\left(x_{0}\right) ; \nabla F\left(x_{0}\right) y\right) .
\end{aligned}
$$

Moreover,

$$
\begin{aligned}
& \partial^{k \infty}\left(f_{1}+f_{2} \circ F\right)\left(x_{0}\right) \subset \partial^{k \infty} f_{1}\left(x_{0}\right)+\nabla F\left(x_{0}\right)^{*} \partial^{k \infty} f_{2}\left(F\left(x_{0}\right)\right) . \\
& \partial^{P}\left(f_{1}+f_{2} \circ F\right)\left(x_{0}\right) \subset \partial^{P} f_{1}\left(x_{0}\right)+\nabla F\left(x_{0}\right)^{*} \partial^{P} f_{2}\left(F\left(x_{0}\right)\right) .
\end{aligned}
$$

Equality holds in (5.3) and (5.5) if $f_{1}^{P}\left(x_{0} ; \cdot\right)=f_{1}^{k}\left(x_{0} ; \cdot\right)$ and

$$
f_{2}^{P}\left(F\left(x_{0}\right) ; \nabla F\left(x_{0}\right)(\cdot)\right)=f^{K}\left(F\left(x_{0}\right) ; \nabla F\left(x_{0}\right)(\cdot)\right),
$$

or if $f_{1}^{P}\left(x_{0} ; \cdot\right)=f_{1}^{K}\left(x_{0} ; \cdot\right)$ and $f_{2}^{P}\left(F\left(x_{0}\right) ; \nabla F\left(x_{0}\right)(\cdot)\right)=f_{2}^{k}\left(F\left(x_{0}\right) ; \nabla F\left(x_{0}\right)(\cdot)\right)$. Replacing " $P$ " with " $k$ " in these conditions gives conditions for equality in (5.2) and (5.4).

Proof. Define $M, G, C$, and $z_{0}$ as in Theorem 4.2. It suffices to prove (2.12). To that end, suppose $w_{n}:=\left(v_{n}, d_{n}\right) \in M(C)$ converges to $M z_{0}=$ $\left(x_{0}, f_{1}\left(x_{0}\right)+f_{2}\left(F\left(x_{0}\right)\right)\right)$ and $t_{n} \downarrow 0$ such that $t_{n}^{-1}\left(w_{n}-M z_{0}\right)$ converges. Then $\left(v_{n}, d_{n}\right)=M\left(x_{n}, y_{n}, a_{n}, r_{n}\right)$ with $f_{1}\left(x_{n}\right) \leq y_{n}, f_{2}\left(a_{n}\right) \leq r_{n}, a_{n}=F\left(x_{n}\right)$, and 
$v_{n}=x_{n}, d_{n}=y_{n}+r_{n}$. Since $t_{n}^{-1}\left(w_{n}-M z_{0}\right)$ converges, it follows that $t_{n}^{-1}\left(x_{1}-x_{0}\right)$ and $t_{n}^{-1}\left(d_{n}-f_{1}\left(x_{0}\right)-f_{2}\left(F\left(x_{0}\right)\right)\right)$ converge. It remains to show that $t_{n}^{-1}\left(y_{n}-f_{1}\left(x_{0}\right)\right)$, $t_{n}^{-1}\left(r_{n}-f_{2}\left(F\left(x_{0}\right)\right)\right)$, and $t_{n}^{-1}\left(a_{n}-F\left(x_{0}\right)\right)$ converge. Since $F$ is strictly differentiable, $t_{n}^{-1}\left(a_{n}-F\left(x_{0}\right)\right)=t_{n}^{-1}\left(F\left(x_{n}\right)-F\left(x_{0}\right)\right)$ converges. Since $f_{1}^{K}\left(x_{0} ; \cdot\right)$ and $\left(f_{2} \circ F\right)^{K}\left(x_{0} ; \cdot\right)$ are proper, the sequences $t_{n}^{-1}\left(y_{n}-f_{1}\left(x_{0}\right)\right)$ and $t_{n}^{-1}\left(r_{n}-f_{2}\left(F\left(x_{0}\right)\right)\right)$ are bounded below by (5.1). If $t_{n}^{-1}\left(y_{n}-f_{1}\left(x_{0}\right)\right)$ were not bounded above, then

$$
t_{n}^{-1}\left(d_{n}-f_{1}\left(x_{0}\right)-f_{2}\left(F\left(x_{0}\right)\right)\right)=t_{n}^{-1}\left(y_{n}-f_{1}\left(x_{0}\right)\right)+t_{n}^{-1}\left(r_{n}-f_{2}\left(F\left(x_{0}\right)\right)\right)
$$

would also not be bounded above, a contradiction. Thus $t_{n}^{-1}\left(y_{n}-f_{1}\left(x_{0}\right)\right)$ is bounded, and taking a subsequence if necessary, we may assume (because of Remark 2.1(b)) that it converges. Then $t_{n}^{-1}\left(r_{n}-f_{2}\left(F\left(x_{0}\right)\right)\right)$ must converge also. We have established (2.12). By Theorem 4.2 with $A=A^{\prime}:=k^{\infty}$ and $A=A^{\prime}=P$, (5.2) through (5.5) hold. The conditions for equality follow from [33, Propositions $6.1,6.2]$.

REMARK 5.2. In the case in which $E=E_{1}$ and $F$ is the identity mapping on $E,(4.6)$ reduces to

$$
\operatorname{dom} f_{1}^{T}\left(x_{0} ;\right)-\operatorname{dom} f_{2}^{T}\left(x_{0} ;\right)=E,
$$

and (5.3) and (5.5) become

$$
\left(f_{1}+f_{2}\right)^{P}\left(x_{0} ; y\right) \leq f_{1}^{P}\left(x_{0} ; y\right)+f_{2}^{P}\left(x_{0} ; y\right)
$$

and

$$
\partial^{P}\left(f_{1}+f_{2}\right)\left(x_{0}\right) \subset \partial^{P} f_{1}\left(x_{0}\right)+\partial f_{2}\left(x_{0}\right),
$$

respectively. Penot [22, Proposition 5.4] has proven (5.7) and (5.8) under different hypotheses. In [22], (5.6) is replaced by

$$
\operatorname{dom} f_{1}^{P}\left(x_{0} ; \cdot\right) \cap \operatorname{dom} f_{2}^{I P}\left(x_{0} ; \cdot\right) \neq \varnothing
$$

where $I P$ is the interiorly prototangent cone

$$
\begin{aligned}
I P_{C}\left(x_{0}\right):=\{y \mid & \forall\left(x_{n}, t_{n}\right) \rightarrow\left(x_{0}, 0^{+}\right) \text {such that } x_{n} \in C \text { and } t_{n}^{-1}\left(x_{n}-x_{0}\right) \\
& \text { converges, } \left.\forall y_{n} \rightarrow y, x_{n}+t_{n} y_{n} \in C \text { for } n \text { sufficiently large }\right\} .
\end{aligned}
$$

Condition (5.9) is sometimes more restictive, sometimes less restrictive, than (5.6). For example, suppose $f_{1}: 1 \mathbf{R}^{2} \rightarrow 1 \mathbf{R}$ and $f_{2}: 1 \mathbf{R}^{2} \rightarrow 1 \mathbf{R}$ are defined by $f_{1}(x, y)=|x|^{1 / 2}$ and $f_{2}(x, y)=|y|^{1 / 2}$. Then at $x_{0}=(0,0), \operatorname{dom} f_{1}^{T}\left(x_{0} ; \cdot\right)=0 \times 1 \mathbf{R}$, $\operatorname{dom} f_{2}^{T}\left(x_{0} ; \cdot\right)=1 \mathbf{R} \times 0$, and $\operatorname{dom} f^{I P}\left(x_{0} ; \cdot\right)=\varnothing$, so that $(5.6)$ holds while $(5.9)$ does not. On the other hand, suppose $f_{i}: 1 \mathbf{R} \rightarrow 1 \mathbf{R}, i=1,2$, are both defined by

$$
f_{i}(x)= \begin{cases}0 & \text { if } x=0 \\ 1 / n & \text { if } 1 /(n+1)<|x| \leq 1 / n, \quad n=1,2,3,4, \ldots, \\ |x| & \text { if }|x|>1\end{cases}
$$

Then $\operatorname{dom} f_{i}^{T}(0 ; \cdot)=\{0\}$ and $\operatorname{dom} f_{i}^{P}(0 ; \cdot)=\operatorname{dom} f_{i}^{I P}(0 ; \cdot)=1 \mathbf{R}$, so that $(5.9)$ holds at $x_{0}=0$ while (5.6) does not.

One advantage of [22, Proposition 5.4] is that it is applicable to functions with general domain spaces. Assumption (5.9) is analogous to

$$
\operatorname{dom} f_{1}^{T}\left(x_{0} ; \cdot\right) \cap \operatorname{int} \operatorname{dom} f_{2}^{T}\left(x_{0} ; \cdot\right) \neq \varnothing,
$$


the assumption under which the sum formula for $f^{T}$ can be proven in general spaces [25, Theorem 2]. Interestingly, while (5.10) can be weakened to (5.6) in finite dimensions [33], we have already seen in Remark 4.7(d) that (5.9) cannot be correspondingly weakened to

$$
\operatorname{dom} f_{1}^{P}\left(x_{0} ; \cdot\right)-\operatorname{dom} f_{2}^{P}\left(x_{0} ; \cdot\right)=E .
$$

We next establish the $A=A^{\prime}:=P, k^{\infty}$ cases of Theorem 4.9, beginning with a technical lemma.

LEMMA 5.3. Let $F: 1 \mathbf{R}^{n} \rightarrow \overline{1 \mathbf{R}}$ be finite at $x_{0}$ and isotone on $N_{\delta}\left(x_{0}\right)$ for some $\delta>0$. Then $F^{k \infty}\left(x_{0} ; \cdot\right)$ and $F^{P}\left(x_{0} ; \cdot\right)$ are isotone on $1 \mathbf{R}^{n}$.

PROOF. Let $y_{1}, y_{2} \in 1 \mathbf{R}^{n}$ with $y_{1} \leq y_{2}$, and suppose that $F^{k \infty}\left(x_{0} ; y_{2}\right) \leq d$. It suffices to show that $F^{k \infty}\left(x_{0} ; y_{1}\right) \leq d$. To this end, let $(z, r) \in k_{F}\left(x_{0}\right)$. Then $\left(z+y_{2}, d+r\right) \in k_{F}\left(x_{0}\right)$. Let $t_{n} \downarrow 0$. There exist $\left(w_{n}, a_{n}\right) \rightarrow(0,0)$ such that $\left(x_{0}, F\left(x_{0}\right)\right)+t_{n}\left(z+y_{2}+w_{n}, d+r_{n}+a_{n}\right) \in$ epi $F$; i.e.,

$$
t_{n}^{-1}\left[F\left(x_{0}+t_{n}\left(z+y_{2}+w_{n}\right)\right)-F\left(x_{0}\right)\right] \leq d+r+a_{n} .
$$

For $n$ large enough, both $x_{0}+t_{n}\left(z+y_{i}+w_{n}\right), i=1,2$, lie in $N_{\delta}\left(x_{0}\right)$. By the isotonicity of $F$, then, $t_{n}^{-1}\left[F\left(x_{0}+t_{n}\left(z+y_{1}+w_{n}\right)\right)-F\left(x_{0}\right)\right] \leq d+r+a_{n}$. Thus $\left(z+y_{1}, d+r\right) \in k_{F}\left(x_{0}\right)$, and it follows that $F^{k \infty}\left(x_{0} ; y_{1}\right) \leq d$. The $A:=P$ case can be proved in a similar fashion.

THEOREM 5.4. Let $f_{i}: E \rightarrow \overline{\mathbf{1 R}}, i=1, \ldots, n$, be finite and Lipschitz-like at $x_{0}$, and define $f:=\left(f_{1}, \ldots, f_{n}\right)$. Let $F: 1 \mathbf{R}^{n} \rightarrow \overline{1 \mathbf{R}}$ be finite at $f\left(x_{0}\right)$, isotone on $N_{\delta}\left(x_{0}\right) \cup B$ for some $\delta>0$, and l.s.c. Assume that (4.14) holds, that each $f_{i}^{K}\left(x_{0} ; \cdot\right)$ is proper, and that $\lim \sup _{k \rightarrow \infty}\left[(F \circ f)\left(x_{0}+t_{k} y_{k}\right)-(F \circ f)\left(x_{0}\right)\right] t_{k}^{-1}=+\infty$ whenever $t_{k} \downarrow 0, y_{k} \rightarrow y$ and $\lim \sup _{k \rightarrow \infty} t_{k}^{-1}\left[f_{i}\left(x_{0}+t_{k} y_{k}\right)-f_{i}\left(x_{0}\right)\right]=+\infty$ for some $i$. Then for all $y \in E$,

$$
\begin{gathered}
(F \circ f)^{k \infty}\left(x_{0} ; y\right) \leq F^{k \infty}\left(f\left(x_{0}\right) ; f_{1}^{k \infty} f\left(x_{0} ; y\right), \ldots, f_{n}^{k \infty}\left(x_{0} ; y\right)\right), \\
(F \circ f)^{P}\left(x_{0} ; y\right) \leq F^{P}\left(f\left(x_{0}\right) ; f_{1}^{P}\left(x_{0} ; y\right), \ldots, f_{n}^{P}\left(x_{0} ; y\right)\right) .
\end{gathered}
$$

Moreover,

$$
\begin{aligned}
\partial^{k \infty}(F \circ f) & \left(x_{0}\right) \\
& \subset\left\{\lambda \cdot\left(\partial^{k \infty} f_{1}\left(x_{0}\right), \ldots, \partial^{k \infty} f_{n}\left(x_{0}\right)\right) \mid \lambda \in \partial^{k \infty} F\left(f\left(x_{0}\right)\right), \lambda \geq 0^{+}\right\} \\
\partial^{P}(F \circ f)\left(x_{0}\right) & \\
& \subset\left\{\lambda \cdot\left(\partial^{P} f_{1}\left(x_{0}\right), \ldots, \partial^{P} f_{n}\left(x_{0}\right)\right) \mid \lambda \in \partial^{P} F\left(f\left(x_{0}\right)\right), \lambda \geq 0^{+}\right\} .
\end{aligned}
$$

Equality holds in (5.14) if $F^{P}\left(f\left(x_{0}\right) ; \cdot\right)=F^{K}\left(f\left(x_{0}\right) ; \cdot\right), f_{1}^{P}\left(x_{0} ; \cdot\right)=f_{1}^{k}\left(x_{0} ; \cdot\right)$, and $f_{i}^{P}\left(x_{0} ; \cdot\right)=f_{i}^{K}\left(x_{0} ; \cdot\right), i=2, \ldots, n$. Equality holds in (5.12) if in addition $F^{K}\left(f\left(x_{0}\right) ; \cdot\right)$ is proper. The replacement of $P$ by $k^{\infty}$ in these conditions gives condition for equality in (5.13) and (5.11).

Proof. Define $M, G, C$, and $z_{0}$ as in Theorem 4.9. It suffices to prove (2.12). Suppose $w_{k}:=\left(v_{k}, d_{k}\right) \in M(C)$ converges to $M z_{0}$ and $t_{k} \downarrow 0$ such that $t_{k}^{-1}\left(w_{k}-M z_{0}\right)$ is convergent. Then

$$
\left(v_{k}, d_{k}\right)=M\left(v_{k}, f_{1}\left(v_{k}\right), \ldots, v_{k}, f_{n}\left(v_{k}\right), f_{1}\left(v_{k}\right), \ldots, f_{n}\left(v_{k}\right), d_{k}\right)
$$


with $d_{k} \geq(F \circ f)\left(v_{k}\right)$ and $y_{k}:=t_{k}^{-1}\left(v_{k}-x_{0}\right)$ and $t_{k}^{-1}\left(d_{k}-(F \circ f)\left(x_{0}\right)\right)$ convergent. It remains to show that $t_{k}^{-1}\left(f_{i}\left(x_{0}+t_{k} y_{k}\right)-f_{i}\left(x_{0}\right)\right)$ converges for each $i$. Each of these sequences is bounded below since $f_{i}^{K}\left(x_{0} ; \cdot\right)$ is proper. Suppose one of them is not bounded above. Then $t_{k}^{-1}\left[(F \circ f)\left(x_{0}+t_{k} y_{k}\right)-(F \circ f)\left(x_{0}\right)\right]$ is also not bounded above, a contradiction of the fact that $t_{k}^{-1}\left[d_{k}-(F \circ f)\left(x_{0}\right)\right]$ converges. Taking a subsequence if necessary, we may assume that $t_{k}^{-1}\left[f_{i}\left(x_{0}+t_{k} y_{k}\right)-f_{i}\left(x_{0}\right)\right]$ converges. Therefore (2.12) holds, and (5.11) through (5.14) follow from Theorem 4.9 and Proposition 2.4. The conditions for equality are a consequence of [33, Proposition 6.1].

Important special cases of Theorem 5.4 include $F\left(z_{1}, \ldots, z_{n}\right)=\sum_{i=1}^{n} z_{i}$ and $F\left(z_{1}, \ldots, z_{n}\right)=\max \left\{z_{1}, \ldots, z_{n}\right\}$. Another corollary of Theorem 5.4 is a product rule for positive-valued functions.

COROLlaRY 5.5. Let $f_{i}: E \rightarrow \overline{1 \mathbf{R}}, i=1, \ldots, n$, be nonnegative on $E$ and Lipschitz-like and positive at $x_{0} \in \operatorname{dom} f_{i}$. Assume that each $f_{i}^{K}\left(x_{0} ; \cdot\right)$ is proper and that $\operatorname{dom} f_{i}^{T}\left(x_{0} ; \cdot\right), i=1, \ldots, n$, are in strong general position. Then for all $y \in E$,

$$
\begin{gathered}
\left(\prod_{i=1}^{n} f_{i}\right)^{k \infty}\left(x_{0} ; y\right) \leq \sum_{i=1}^{n}\left(\prod_{j \neq i} f_{j}\left(x_{0}\right)\right) f_{i}^{k \infty}\left(x_{0} ; y\right), \\
\left(\prod_{i=1}^{n} f_{i}\right)^{P}\left(x_{0} ; y\right) \leq \sum_{i=1}^{n}\left(\prod_{j \neq i} f_{j}\left(x_{0}\right)\right) f_{i}^{P}\left(x_{0} ; y\right) .
\end{gathered}
$$

Moreover,

$$
\begin{gathered}
\partial^{k \infty}\left(\prod_{i=1}^{n} f_{i}\right)\left(x_{0}\right) \subset \sum_{i=1}^{n}\left(\prod_{j \neq 1} f_{j}\left(x_{0}\right)\right) \partial^{k \infty} f_{i}\left(x_{0}\right) . \\
\partial^{P}\left(\prod_{i=1}^{n} f_{i}\right)\left(x_{0}\right) \subset \sum_{i=1}^{n}\left(\prod_{j \neq i} f_{j}\left(x_{0}\right)\right) \partial^{P} f_{i}\left(x_{0}\right) .
\end{gathered}
$$

Equality holds in (5.16) and (5.18) if $f_{i}^{P}\left(x_{0} ; \cdot\right)=f_{i}^{k}\left(x_{0} ; \cdot\right)$ for some $i$ and $f_{j}^{P}\left(x_{0} ; \cdot\right)$ $=f_{j}^{K}\left(x_{0} ; \cdot\right)$ for each $j \neq i$. The replacement of $P$ by $k^{\infty}$ in these conditions gives conditions for equality in (5.15) and (5.17).

Proof. In Theorem 5.4, define $F: 1 \mathbf{R}^{n} \rightarrow 1 \mathrm{R}$ by $f\left(z_{1}, \ldots, z_{n}\right)=\prod_{i=1}^{n} z_{i}$. Condition (4.14) in this case reduces to $\operatorname{dom} f_{i}^{T}\left(x_{0} ;\right), i=1, \ldots, n$, being in strong general position. To verify the remaning hypothesis of Theorem 5.4, suppose $t_{k} \downarrow 0$ and $y_{k} \rightarrow y$. Since each $f_{i}$ is strictly l.s.c. at $x_{0}$, for each $\delta>0$ there exists $m$ such that for all $k \geq m, f_{i}\left(x_{0}+t_{k} y_{k}\right) \geq f_{i}\left(x_{0}\right)-\delta$. Let $\varepsilon>0$ be given. Then there exists $n_{0}$ such that for all $k \geq n_{0}$,

$$
\begin{aligned}
& t_{k}^{-1}\left[(F \circ f)\left(x_{0}+t_{k} y_{k}\right)-(F \circ f)\left(x_{0}\right)\right] \\
& \quad \geq \sum_{i=1}^{n}\left(\prod_{j \neq i} f_{j}\left(x_{0}\right)\right) t_{k}^{-1}\left[f_{i}\left(x_{0}+t_{k} y_{k}\right)-f_{i}\left(x_{0}\right)\right]-\varepsilon .
\end{aligned}
$$


If for some $i, \lim \sup _{k \rightarrow \infty} t_{k}^{-1}\left[f_{i}\left(x_{0}+t_{k} y_{k}\right)-f_{i}\left(x_{0}\right)\right]=+\infty$, it follows from the above inequality that

$$
\limsup _{k \rightarrow \infty} t_{k}^{-1}\left[(F \circ f)\left(x_{0}+t_{k} y_{k}\right)-(F \circ f)\left(x_{0}\right)\right]=+\infty
$$

Thus the hypotheses of Theorem 5.4 are satisfied and (5.15) through (5.18) follow from (5.14).

Some calculus formulae for $f^{K \infty}$ can be proven by means of our results for the $A:=K, A^{\prime}:=k$ case.

Proposition 5.6. Let $F: E \rightarrow E_{1}$ be strictly differentiable on some $N_{\delta}\left(x_{0}\right)$ and $f: E_{1} \rightarrow \overline{\mathbf{1 R}}$ Lipschitz-like and finite at $F\left(x_{0}\right)$. Assume

$$
\nabla F\left(x_{0}\right) E-\operatorname{dom} f^{T}\left(x_{0} ;\right)=E_{1} .
$$

Then for all $y \in E$,

$$
(f \circ F)^{K \infty}\left(x_{0} ; \dot{y}\right)=f^{K \infty}\left(F\left(x_{0}\right) ; \nabla F\left(x_{0}\right) y\right) .
$$

Moreover,

$$
\partial^{K \infty}(f \circ F)\left(x_{0}\right)=\nabla F\left(x_{0}\right)^{*} \partial^{K \infty} f\left(F\left(x_{0}\right)\right) .
$$

ProOF. The inequality $(f \circ F)^{K}\left(x_{0} ; y\right) \geq f^{K}\left(F\left(x_{0}\right) ; \nabla F\left(x_{0}\right) y\right)$ is true in general [33, Proposition 6.2], and $(f \circ F)^{K}\left(x_{0} ; y\right) \leq f^{K}\left(F\left(x_{0}\right) ; \nabla F\left(x_{0}\right) y\right)$ holds under assumption (5.19) by Theorem 4.2 (and Remark 4.3(c)) with $A:=K, A^{\prime}:=k$, and $f_{1} \equiv 0$. Thus $(5.20)$ holds. Since $T \subset K^{\infty},(5.19)$ implies that $\nabla F\left(x_{0}\right) E-$ $\operatorname{dom} f^{K \infty}\left(x_{0} ; \cdot\right)=E_{1}$. Equation (5.21) then follows from (5.20) and the corresponding convex analysis result.

THEOREM 5.7. Let $f_{1}: E \rightarrow \overline{\mathbf{1 R}}$ and $f_{2}: E \rightarrow \overline{\mathbf{R}}$ be finite and Lipschitz-like at $x_{0}$, and suppose (5.6) holds. Assume in addition that $f_{2}^{K}\left(x_{0} ; \cdot\right)=f_{2}^{k}\left(x_{0} ; \cdot\right)$ and that $f_{1}^{K}\left(x_{0} ; \cdot\right)$ and $f_{2}^{K}\left(x_{0} ; \cdot\right)$ are proper. Then for all $y \in E$,

$$
\left(f_{1}+f_{2}\right)^{K \infty}\left(x_{0} ; y\right) \leq f_{1}^{K \infty}\left(x_{0} ; y\right)+f_{2}^{K \infty}\left(x_{0} ; y\right) \text {. }
$$

Moreover,

$$
\partial^{K \infty}\left(f_{1}+f_{2}\right)\left(x_{0}\right) \subset \partial^{K \infty} f_{1}\left(x_{0}\right)+\partial^{K \infty} f_{2}\left(x_{0}\right) .
$$

Equality holds in (5.22) and (5.23) if in addition $f_{i}^{K \infty}\left(x_{0} ; \cdot\right)=f_{i}^{K}\left(x_{0} ; \cdot\right), i=1,2$.

ProOF. Let $\left(y, r_{i}\right) \in K_{f_{i}}^{\infty}\left(x_{0}\right), i=1,2$. To prove (5.22), it suffices to show that $\left(y, r_{1}+r_{2}\right) \in K_{f_{1}+f_{2}}^{\infty}\left(x_{0}\right)$. Suppose $(z, s) \in K_{f_{1}+f_{2}}\left(x_{0}\right)$. Since $f_{1}^{K}\left(x_{0} ; \cdot\right)$ and $f_{2}^{K}\left(x_{0} ;\right)$ are proper, there exist $s_{1}, s_{2} \in 1 \mathbf{R}$ such that $s=s_{1}+s_{2}$ and $\left(z, s_{i}\right) \in$ $K_{f_{i}}\left(x_{0}\right)$. Then $\left(y+z, r_{i}+s_{i}\right) \in K_{f_{i}}\left(x_{0}\right)$. By hypothesis, $K_{f_{2}}\left(x_{0}\right)=k_{f_{2}}\left(x_{0}\right)$. Apply Theorem 4.2 with $E=E_{1}, A:=K, A^{\prime}:=k$, and $F$ the identity mapping on $E$ to deduce that $\left(y+z, r_{1}+r_{2}+s\right) \in K_{f_{1}+f_{2}}\left(x_{0}\right)$. Therefore $\left(y, r_{1}+r_{2}\right) \in K_{f_{1}+f_{2}}^{\infty}\left(x_{0}\right)$, and the proof of (5.22) is complete. Inclusion (5.23) follows from (5.22) and [17, $1.2 .5]$

An analogue of Theorem 5.4 for $K^{\infty}$ can be derived by the above method under the assumption that $f_{i}^{K}\left(x_{0} ; \cdot\right)=f_{i}^{k}\left(x_{0} ; \cdot\right), i=2, \ldots, n$. Details are left to the reader. 
6. Necessary conditions for optimality. The case $A:=K, A^{\prime}:=k$ in Theorem 4.2 can be applied to prove quite general necessary conditions for local optimality in the abstract mathematical program

$$
\min \{f(x) \mid x \in C\} .
$$

Our results will rely on the fact that if $x_{0}$ is an unconstrained local minimizer for $f: E \rightarrow \overline{\mathbf{1 R}}$, then $f^{K}\left(x_{0} ; y\right) \geq 0$ for all $y \in E$ (see for example [26]). We begin with a refinement of optimality conditions given in $[21$, Proposition $4.1 ; 13$, Theorem 5 ; 34, Corollary 3.3].

THEOREM 6.1. Let $C \subset E$ be epi-Lipschitz-like at $x_{0} \in C$, and suppose $f: E \rightarrow$ $\overline{1 \mathbf{R}}$ is finite and Lipschitz-like at $x_{0}$, a local minimizer for $(\mathrm{P})$. Assume

$$
\operatorname{dom} f^{T}\left(x_{0} ; \cdot\right)-T_{C}\left(x_{0}\right)=E .
$$

Then

$$
\begin{aligned}
& f^{K}\left(x_{0} ; y\right) \geq 0 \quad \text { for all } y \in k_{C}\left(x_{0}\right) \\
& f^{k}\left(x_{0} ; y\right) \geq 0 \quad \text { for all } y \in K_{C}\left(x_{0}\right)
\end{aligned}
$$

PROOF. The point $x_{0}$ is an unconstrained local minimizer for the function $f+i_{C}$. Hence for all $y \in E, 0 \leq\left(f+i_{C}\right)^{K}\left(x_{0} ; y\right)$. Assumption (6.1) allows us to apply Theorem 4.2 with $A:=K, A^{\prime}:=k, E=E_{1}$ and $F$ the identity mapping $E$ to obtain

$$
\begin{gathered}
0 \leq f^{K}\left(x_{0} ; y\right)+i_{C}^{k}\left(x_{0} ; y\right), \\
0 \leq f^{k}\left(x_{0} ; y\right)+i_{C}^{K}\left(x_{0} ; y\right)
\end{gathered}
$$

for all $y \in E$. Now if $y \in k_{C}\left(x_{0}\right), i_{C}^{k}\left(x_{0} ; y\right)=0$ and (6.2) follows from the first of these inequalities. Condition (6.3) follows in like manner from the second inequality.

REMARK 6.2. Theorem 6.1 generates a whole family of necessary conditions. If $A \subset K$ and $A^{\prime} \subset k$ are tangent cones,

$$
\begin{aligned}
& f^{A}\left(x_{0} ; y\right) \geq 0 \quad \text { for all } y \in A_{C}^{\prime}\left(x_{0}\right), \\
& f^{A^{\prime}}\left(x_{0} ; y\right) \geq 0 \quad \text { for all } y \in A_{C}\left(x_{0}\right)
\end{aligned}
$$

are necessary conditions for local optimality in $(\mathrm{P})$ under assumption (6.1). In other words, Theorem 6.1 expands the class of "upper convex approximants" [23, $\mathbf{1 4}, \mathbf{3 4}]$ or "approximate quasidifferentials" [16] for which optimality conditions can be stated. The cases $A=A^{\prime}:=T, P$, or $k^{\infty}$ can of course be alternately derived from sum formulae for $f^{T}, f^{P}$, and $f^{k \infty}$.

One important special case of problem (P) is that in which $C:=\left\{x \mid g_{i}(x) \leq\right.$ $0, i=1, \ldots, n\}$, the set of points satisfying a finite number of inequality constraints. Tangent cones of such sets have been calculated (with the help of special cases of Proposition 4.6) in [33 and 34]. We list the basic result below.

Proposition $6.3[\mathbf{3 3}, \mathbf{3 4}]$. Let $g: E \rightarrow \overline{\mathbf{1 R}}$ be Lipschitz-like at $x_{0} \in g^{-1}(0)$. Suppose there exists $y \in E$ with $g^{T}\left(x_{0} ; y\right)<0$ (or equivalently, that $0 \notin \partial^{T} g\left(x_{0}\right)$ ). Define $C:=\{x \in E \mid g(x) \leq 0\}$. Then

$$
\begin{aligned}
& K_{C}\left(x_{0}\right)=\left\{y \in E \mid g^{K}\left(x_{0} ; y\right) \leq 0\right\}, \\
& k_{C}\left(x_{0}\right)=\left\{y \in E \mid g^{k}\left(x_{0} ; y\right) \leq 0\right\} \\
& T_{C}\left(x_{0}\right) \supset\left\{y \in E \mid g^{T}\left(x_{0} ; y\right) \leq 0\right\} .
\end{aligned}
$$


Proposition 6.4. Let $f: E \rightarrow \overline{1 \mathbf{R}}$ and $g_{i}: E \rightarrow \overline{1 \mathbf{R}}, i=1, \ldots, n$, be Lipschitzlike at $x_{0}$, a local minimizer for

$$
\min \left\{f(x) \mid g_{i}(x) \leq 0, i=1, \ldots, n\right\} .
$$

Define $I\left(x_{0}\right):=\left\{j \mid g_{j}\left(x_{0}\right)=0\right\}$. Assume tht $g_{j}$ is continuous at $x_{0}$ for each $j \notin I\left(x_{0}\right)$, that $0 \notin \partial^{T} g_{j}\left(x_{0}\right)$ for each $j \in I\left(x_{0}\right)$, and that $\operatorname{dom} f^{T}\left(x_{0} ; \cdot\right)$ and $\left\{y \mid g_{j}^{T}\left(x_{0} ; y\right) \leq 0\right\}, j \in I\left(x_{0}\right)$, are in strong general position. Then

$$
\begin{gathered}
f^{K}\left(x_{0} ; y\right) \geq 0 \quad \text { whenever } g_{j}^{k}\left(x_{0} ; y\right) \leq 0 \text { for all } j \in I\left(x_{0}\right), \\
f^{k}\left(x_{0} ; y\right) \geq 0 \quad \text { whenever } g_{i}^{K}\left(x_{0} ; y\right) \leq 0 \text { for some } i \in I\left(x_{0}\right) \text { and } \\
\qquad g_{j}^{k}\left(x_{0} ; y\right) \leq 0 \text { for all } j \in I\left(x_{0}\right) \backslash\{i\} .
\end{gathered}
$$

ProOF. Let $C:=\left\{x \mid g_{j}\left(x_{0}\right) \leq 0, j=1, \ldots, n\right\}$ in problem (P), and call $D_{j}:=\left\{x \mid g_{j}\left(x_{0}\right) \leq 0\right\}$. For $j \notin I\left(x_{0}\right), T_{D_{j}}\left(x_{0}\right)=E$ since $g_{j}$ is continuous at $x_{0}$. By (6.8), $T_{D_{j}}\left(x_{0}\right), j=1, \ldots, n$, are in strong general position, so we may apply Proposition 4.6 with $A=A^{\prime}:=T$ to deduce that

$$
T_{C}\left(x_{0}\right) \supset\left\{y \mid g_{j}^{T}\left(x_{0} ; y\right) \leq 0, j \in I\left(x_{0}\right)\right\} .
$$

This inclusion and our strong general position assumption imply that (6.1) holds. Then (6.10) and (6.11) follow from (6.2), (6.3) and Proposition 4.6.

One can also derive optimality conditions in the form of subgradient inclusions for problem (6.9).

THEOREM 6.5. Let $x_{0}$ be a local minimizer for (6.9), and suppose $A$ is a convex tangent cone satisfying (1.2) and $A_{j}, j \in I\left(x_{0}\right)$, are convex tangent cones such that $T \subset A_{j} \subset k$. In addition to the hypotheses of Proposition 6.4, assume that $\partial^{A j} g_{j}\left(x_{0}\right) \notin \varnothing$ for each $j \in I\left(x_{0}\right)$. Then there exist $\lambda_{j} \geq 0, j \in I\left(x_{0}\right)$, such that

$$
0 \in \partial^{A} f\left(x_{0}\right)+\sum_{I\left(x_{0}\right)}\left(\lambda_{j} \partial^{A j} g_{j}\left(x_{0}\right) \cup 0^{+} \partial^{A j} g_{j}\left(x_{0}\right)\right) \text {. }
$$

Proof. Define $C$ and $D_{j}, j=1, \ldots, n$, as in Proposition 6.4. As in the proof of Theorem 6.1, for all $y \in E$

$$
0 \leq f^{K}\left(x_{0} ; y\right)+i_{C}^{k}\left(x_{0} ; y\right)=f^{K}\left(x_{0} ; y\right)+\sum_{I\left(x_{0}\right)} i_{D_{j}}^{k}\left(x_{0} ; y\right)
$$

since $k_{C}\left(x_{0}\right)=\bigcap_{I\left(x_{0}\right)} k_{D_{j}}\left(x_{0}\right)$ by Proposition 4.6. Now call $S_{j}=\left\{x \mid g_{j}^{A j}\left(x_{0} ; y\right) \leq\right.$ $0\}$ for $j \in I\left(x_{0}\right)$. By $(6.7), S_{j} \subset k_{D_{j}}\left(x_{0}\right)$. Thus $0 \leq f^{A}\left(x_{0} ; y\right)+\sum_{I\left(x_{0}\right)} i_{S_{j}}(y)$ for all $y \in E$. It follows that

$$
0 \in \partial\left(f^{A}\left(x_{0} ; \cdot\right)+\sum_{I\left(x_{0}\right)} i_{S_{j}}(\cdot)\right)(0)=\partial^{A} f\left(x_{0}\right)+\sum_{I\left(x_{0}\right)} S_{j}^{0}
$$

since our strong general position assumption allows us to apply [17, Theorem 1.2.5]. By [24, Theorems 23.7, 9.6], (6.12) holds.

The subdifferential calculus developed here and in [33] also enables one to handle objective functions $f$ of various forms - for example, $f:=g \circ G$, where $G$ is strictly differentiable ([14] shows the importance of this form); $f:=g / h$, where $g$ and $h$ are positive-valued and $h$ is continuous [33]; and $f:=\max _{1 \leq i \leq n} h_{i}$. 
7. Conclusions. We have demonstrated that two tangent cones, $k^{\infty}$ and $P$, which can give a sharper local approximation to a set than the Clarke tangent cone $T$, have as strong a subdifferential calculus as $T$. We have also shown that any pair of convex tangent cones $A$ and $A^{\prime}$ with $T \subset A \subset K, T \subset A^{\prime} \subset k$ can be used in place of $T$ in necessary optimality conditions. Nevertheless, the hypotheses in our results seem to necessarily involve $T$ (as do those in [15]), which suggests that the Clarke tangent cone occupies a unique and essential position in the theory of nonsmooth analysis. In summary, no one tangent cone possesses all desirable properties; the most complete theory of nonsmooth analysis and optimization combines several tangent cones.

ACKNOWLEDGMENTS I would like to thank J. M. Borwein, who introduced me to the subject of this paper, and J.-P. Penot, who provided important background material.

\section{REFERENCES}

1. J.-P. Aubin and I. Ekeland, Applied nonlinear analysis, Wiley, New York, 1984.

2. J. M. Borwein, Stability and regular points of inequality systems, J. Optim. Theory Appl. 48 (1986), 9-52.

3. J. M. Borwein, Epi-Lipschitz-like sets in Banach space: theorems and examples, Nonlinear Anal. (to appear).

4. J. M. Borwein and H. M. Strojwas, The hypertangent cone study (submitted).

5. F. H. Clarke, Optimization and nonsmooth analysis, Wiley, New York, 1983.

6. S. Dolecki, Tangency and differentiation: some applications of convergence theory, Ann. Mat. Pura Appl. 130 (1982), 223-255.

7. H. Frankowska, Inclusions adjointes associés aux trajectoires minimales d'inclusions différentielles, C.R. Acad. Sci. Paris 297 (1983), 461-464.

8. __ Necessary conditons for the Bolza problem, Math. Oper. Res. 10 (1985), 361-366.

9. E. Giner, Ensembles et fonctions étoilés. Application au calcul différentiel généralisé, Univ. Toulouse III, 1981, Manuscript.

10. M. Guignard, Generalized Kuhn-Tucker conditions for mathematical programming in a Banach space, SIAM J. Control 7 (1969), 232-241.

11. J.-B. Hiriart-Urruty, Contributions à la programmation mathématique: deterministe et stochastique, Thése, Univ. de Clermont-Ferrand II, 1977.

12. _ , Tangent cones generalized gradients and mathematical programming in Banach spaces, Math. Oper. Res. 4 (1979), 79-97.

13. A. Ioffe, Regular points of Lipschitz mappings, Trans. Amer. Math. Soc. 251 (1979), 61-69.

14. __ Necessary and sufficient conditions for a local minimum, SIAM J. Control Optim. 17 (1979), 245-288.

15. __ Approximate subdifferentials and applications. I: The finite-dimensional theory, Trans. Amer. Math. Soc. 281 (1984), 389-416.

16. V. Jeyakumar, On optimality conditions in nonsmooth inequality constrained minimization, Univ. of Melbourne, Research Report No. 13, 1985.

17. A. G. Kusraev and G. G. Kutateladze, Local convex analysis, J. Soviet Math. 26 (1984), 2048-2087.

18. D. H. Martin, R. J. Gardner, and G. G. Watkins, Indicating cones and the intersection principle for tangential approximants in abstract multiplier rules, Optim. Theory Appl. 33 (1981), 515537.

19. D. H. Martin and G. G. Watkins, Cores of tangent cones and Clarke's tangent cone, Math. Oper. Res. 10 (1985), 565-575.

20. P. Michel and J.-P. Penot, Calcul sous-différentiel pour des fonctions lipschitziennes et non lipschitziennes, C. R. Acad. Sci. Paris 298 (1984), 269-272.

21. J.-P. Penot, Calcul sous-différentiel et optimisation, J. Funct. Anal. 27 (1978), 248-276. 
22. __ Variations on the theme of nonsmooth analysis: another subdifferential, (V. F. Demyanov and D. Pallaschke, eds.), Nondifferentiable Optimization: Motivations and Applications, Lecture Notes in Economics and Mathematical Systems, vol. 255, Springer-Verlag, Berlin and New York, 1985.

23. B.N. Pshenichnyi and R. A. Khachatryan, Constraints of equality tupe in nonsmooth optimization problems, Soviet Math. Dokl. 26 (1982), 659-662.

24. R. T. Rockafellar, Convex analysis, Princeton Univ. Press, Princeton, N. J., 1970.

25. __ Directionally Lipschitzian functions and subdifferential calculus, Proc. London Math. Soc. 39 (1979), 331-355.

26. _ - The theory of subgradients and its application to problems of optimization: Convex and nonconvex functions, Heldermann Verlag, Berlin, 1981.

27. __ Lagrange multipliers and subderivatives of optimal value functions in nonlinear programming, Math. Programming Stud. 17 (1982), 28-66.

28. __ Extensions of subgradient calculus with application to optimization, Nonlinear Anal. 9 (1985), 665-698.

29. J. S. Treiman, Shrinking the set of generalized gradients (submitted).

30. C. Ursescu, Tangent sets' calculus and necessary conditions for extremality, SIAM J. Control Optim. 20 (1982), 563-574.

31. M. Vlach, Approximation operators in optimization theory, Z. Oper. Res. Ser. A-B 25 (1981), 15-23.

32. D. E. Ward, Tangent cones, generalized subdifferential calculus, and optimization, Thesis, Dalhousie Univ. 1984.

33. D. E. Ward and J. M. Borwein, Nonsmooth calculus in finite dimensions, SIAM J. Control Optim. (to appear).

34. D. E. Ward, Isotone tangent cones and nonsmooth optimization, Miami Univ. preprint, 1986.

35. G. G. Watkins, Nonsmooth Milyutin-Dubovitskii theory and Clarke's tangent cone, Math. Oper. Res. 11 (1986), 70-80.

36. C. Zalinescu, On convex sets in general position, Linear Algebra Appl. 34 (1985), 191-198.

Department of Mathematics and Statistics, Miami University, Oxford, Ohio 45056 